\title{
Analysis of Blocking in mmWave Cellular Systems: Application to Relay Positioning
}

\author{
Cristian García Ruiz ${ }^{\circledR}$, Antonio Pascual-Iserte ${ }^{\circledR}$, Senior Member, IEEE, and Olga Muñoz ${ }^{\circledR}$, Member, IEEE
}

\begin{abstract}
Within the framework of 5G, blockage effects occurring in the mmWave band are critical. Previous works describe the effects of blockages in isolated and multiple links for simple blocking objects, modeled with mathematical tools such as stochastic geometry and random shape theory. Our study uses these tools to characterize a scenario with $N$ links, including the possible correlation among them in terms of blocking for several models of blocking objects. We include numerical evaluations highlighting that assuming independence among the links' blocking elements is a too-brief simplification and does not accurately describe the real scenario. This paper also applies the formulation developed for the case of $N$ links to optimize the relay positioning in mmWave cells for coverage enhancement, that is, to minimize the communication failure probability. We also show that both link budget and blockages affect the optimum positioning of the relays as they are both essential for successful transmission.
\end{abstract}

Index Terms-mmWave, blockage, stochastic geometry, random shape theory, relay.

\section{INTRODUCTION}

\section{A. Background and Motivation}

$\mathbf{U}$ SERS demand day after day much faster, higher capacity, and broader coverage in mobile communications. A high number of new social applications and the growing interest in the mobile market has acted as a catalyst in this field. Existing technologies, including long term evolution (LTE), have served well for many years but, since some time ago, it is clear that a new generation of mobile communications is needed. Some applications are just taking off, requiring higher quality standards than previous generations. An example is an increasing interest in autonomous cars that have to share information in real-time with each other to make autonomous driving possible. Communications have to support high traffic,

Manuscript received February 6, 2020; revised July 29, 2020; accepted November 9, 2020. Date of publication November 16, 2020; date of current version February 17, 2021. The work presented in this paper has been funded through the projects 5G\&B-RUNNER-UPC (Agencia Estatal de Investigación and Fondo Europeo de Desarrollo Regional, TEC2016-77148-C2-1-R / AEI/FEDER, UE) and ROUTE56 (Agencia Estatal de Investigación, PID2019104945GB-I00 / AEI / 10.13039/501100011033); and the grant 2017 SGR 578 (Catalan Government-Secretaria d'Universitats i Recerca, Departament d'Empresa i Coneixement, Generalitat de Catalunya, AGAUR). The associate editor coordinating the review of this article and approving it for publication was W. Chen. (Corresponding author: Antonio Pascual-Iserte.)

The authors are with the Department of Signal Theory and Communications, Universitat Politècnica de Catalunya, 08034 Barcelona, Spain (e-mail: cristian.garcia.ruiz@estudiantat.upc.edu; antonio.pascual@upc.edu; olga.munoz@upc.edu).

Color versions of one or more figures in this article are available at https://doi.org/10.1109/TCOMM.2020.3038177.

Digital Object Identifier 10.1109/TCOMM.2020.3038177 secure, and almost instantaneous information transmission, as explained in [1]. As another example, the Internet of Things (IoT) raises the need to support very dense networks. These are just some of the many reasons why the technological community is adopting the new communications standard known as $5 \mathrm{G}[2]$.

In order to meet the requirements exposed above, the usage of mmWave bands (that is, frequencies above $6 \mathrm{GHz}$ ) becomes crucial [3]. The main reason for this is that mmWave allows larger bandwidths, which results in higher data rates, as explained in [4], although mmWave bands also present several significant adverse effects. The first one is the high attenuation and penetration losses compared with lower frequencies used in previous standards. Another effect is the harmful diffraction of the electromagnetic waves [1] because the wavelength is typically smaller than the sizes of objects in the environment. The main consequence is that any object with an electric size higher than the wavelength (which frequently happens due to the small wavelength) will block the signal propagation. Because of the very high penetration and diffraction losses in mmWave channels [5], the direct path contains almost all the energy, and nearly no other multipath components exist. Therefore, line of sight (LOS) conditions mainly decide the performance of mmWave systems [6], [7]. In other words, in mmWave signal transmission, having a successful transmission requires LOS, that is, no blockage between the transmitter and the receiver.

Due to the previous reasons, we need a framework to statistically model blockages and a proper strategy to improve coverage. This is the motivation for this work. The starting point is based on the previous papers [8]-[10] that use stochastic geometry [11]-[13] to model the number of blocking elements and their positions. Blockages are assumed to be uniformly distributed in space following a Poisson point process (PPP). The blocking elements (e.g., buildings) may have different shapes. Several models of blocking elements are considered: line segments and rectangles (with and without height), whose sizes and orientations are modeled as random through random shape theory [14]. These papers obtain the probability of blockage for specific and isolated links. For multiple links, the assumption that the blockages on each of them are independent might be inaccurate. For instance, if the angle between a pair of links is small, the links will likely have some blockages in common. This fact is shown in [6], [15], [16], in which the correlation between the blockings of different links is considered but only for the case of line 
segments. In all the works referenced previously, the positions of the transmitters and the receivers are non-random and known.

In [17], the authors consider the concrete case of rectangles whose side lengths are Gaussian distributed. Height is incorporated using the same procedure as in [9]. However, that procedure is only valid for line segments with height, but not for volumes. The authors also consider multiple links to several access points, but do not take into account the possible blocking correlation among links. In [10], the authors consider a single-link and model blocking objects as rectangles with height. An interesting contribution of [10] is the consideration of the Fresnel zone to derive the stochastic frequency-dependent LOS probability of the link. They compare this probability with the visual LOS probability, i.e., the probability of no blockages intersecting with the visual sightline between the transmitter and the receiver, which is independent of the frequency. The results in [10] show that the visual LOS probability is an accurate estimation of the LOS probability (the error is less than $1 \%$ for frequencies above $20 \mathrm{GHz}$ ). As it is simpler to compute, in this paper we will focus on the visual LOS probability (from now on referred to as the LOS probability).

A way to improve coverage is through the use of relays. In [18], the authors consider a network with a transmitter and a receiver at given positions and relays at random positions. They analyze the performance for this network without incorporating the correlation among the blockages in different user-relay links. In [19], the authors consider a network with one transmitter and several nodes at concrete static positions. Whenever a node is blocked, it can connect to another one that takes the role of a relay. The node selected for relaying is that having the lowest probability of being blocked. In that paper, the blocking elements are modeled as a PPP with circles having given radius and without height, which avoids the need for using random shape theory.

In this paper, we make a statistical analysis of the effects of blockages in the scenario of multiple links without considering independence among them, as done in [6] and [15], but considering more general blocking object shapes. As an example of application, we use this analysis to optimize the positions of a set of relays in a mobile cell to minimize the impact of blockage, improving network coverage.

Another aspect to consider in mmWave signal transmission is the atmospheric attenuation, mainly due to rain [20]-[22]. The authors of [20] propose to compute the frequency-dependent rain attenuation factor as an increasing function of the carrier frequency and the distance between transmitter and receiver, and present experimental measurements for LOS mmWave links in Beijing, China. The authors in [21] aim to retrieve the path-averaged rain rate from LOS mmWave link measurements to assist rainfall monitoring. They also estimate the variance of the local fading (1.65 for the measured LOS link). In [22], the authors also discuss rain attenuation and atmospheric absorption. Although the rain attenuation and oxygen absorption in the 60 and $73 \mathrm{GHz}$ bands are significant (see Table 2 in [22]), the 28 and $38 \mathrm{GHz}$ bands suffer from low rain attenuation and oxygen absorption
( 0.18 and $0.9 \mathrm{~dB}$ for $5 \mathrm{~mm} / \mathrm{h}$ and $25 \mathrm{~mm} / \mathrm{h}$ rain, respectively, and $0.04 \mathrm{~dB}$ of oxygen absorption).

Our primary focus is to study the blocking effects produced by objects within the LOS between the transmitter and the receiver and the losses produced by distance. Note that, to account for fading and atmospheric effects, we could add a random term in the signal losses or consider an augmented sensitivity in the receiver to prevent link failure due to additional signal losses produced by these effects. This inclusion requires a precise and detailed statistical analysis of the fading and atmospheric effects that falls out of the scope of this paper, and we leave it for future research work.

\section{B. Contributions}

The main contributions of this paper with respect to (w.r.t.) the works referenced previously are the following:

- Consider multiple links and take into account the correlation among the blockages in these links for the most general model of blocking elements (that is, rectangles with height).

- Apply the obtained formulation to the mobile cell scenario and use it to design a relay-based network deployment. The blockage probability without assuming independence among the links is formulated for a relay-system and averaged over any possible position of the user within the cell, providing a global figure of merit of the cell.

- Find the optimum positions of the relays to minimize the average probability of not having successful transmission (that is, communication failure probability), where the average is taken w.r.t. to both the randomness of the blocking elements and the random position of the user. In this sense, not only the blockages but also the link budget and sensitivity parameters are considered in the optimization of the relay positioning.

\section{Organization}

The paper is organized as follows. In Section II, we provide the blockage probability in a single-link scenario for different blockage models. In Section III, we extend the previous analysis to a scenario with multiple links and derive the formulation of the probability of having successful transmission. In Section IV, we apply the results obtained in previous sections to the analysis of relay-based communications in terms of the probability of coverage averaged over the random position of the user. We take into account parameters related to power and sensitivity as well. A comparison between simulation results and the analytical expressions derived in this work can be found in Section V. Finally, conclusions are detailed in Section VI.

\section{Notation}

In this paper, we use the symbols and notation detailed in Table I. 
TABLE I

SYMBOLS AND NOTATION

\begin{tabular}{|c|c|}
\hline Notation & Description \\
\hline$S \subset \mathbb{R}^{n}$ & Set or region $S$ in $\mathbb{R}^{n}$ \\
\hline $\mathbb{1}_{S}(\boldsymbol{x}):=\left\{\begin{array}{lll}1 & \text { if } & \boldsymbol{x} \in S \\
0 & \text { if } & \boldsymbol{x} \notin S\end{array}\right.$ & Indicative function \\
\hline$A_{S}=\int \mathbb{1}_{S}(\boldsymbol{x}) d \boldsymbol{x}$ & Area (i.e., size) of region $S \subset \mathbb{R}^{2}$ \\
\hline $\mathbb{P}(\cdot), \bar{X}=\mathbb{E}[X]$ & Probability operator, mean value of random variable (r.v.) $X$ \\
\hline$K \sim \mathcal{P}(\bar{K}), X \sim \mathcal{U}[a, b]$ & $K$ is a Poisson r.v. with mean value $\bar{K}=\mathbb{E}[K], X$ is a uniform r.v. in $[a, b]$ \\
\hline$\vee, \wedge, \cup, \cap$ & Logical 'or' and 'and' operators, union and intersection of sets \\
\hline$L, W, H, \Theta$ & R.v.'s representing the lengths, widths, heights, and orientations of the blocking objects \\
\hline$f_{L}(l), f_{W}(w), f_{H}(h), f_{\Theta}(\theta)$ & Probability density functions (pdf's) of the r.v.'s $L, W, H, \Theta$ \\
\hline$l, w, h, \theta$ & Realizations of the r.v.'s $L, W, H, \Theta$ \\
\hline$L_{\max }, W_{\max }, H_{\max }, \Theta_{\max }$ & Maximum values of $l, w, h, \theta$ \\
\hline$K_{l w h \theta}, \bar{K}_{l w h \theta}=\mathbb{E}\left[K_{l w h \theta}\right]$ & Number of objects (with parameters $l, w, h, \theta$ ) producing blocking, average value \\
\hline$K, \bar{K}=\mathbb{E}[K]$ & Total number of objects producing blocking, average value \\
\hline$S_{l w h \theta}$ & Region containing centers of objects (with parameters $l, w, h, \theta$ ) producing blocking \\
\hline$\lambda_{l w h \theta}$ & Differential spatial density of potential blocking objects with parameters $l, w, h, \theta$ \\
\hline$\lambda$ & Spatial density of potential blocking objects with any size and orientation \\
\hline$H_{0}, H_{1}, H_{B}, H_{U}$ & Heights of transmitter, receiver, base station (BS), user equipment (UE) \\
\hline$O K_{i}, K O_{i}, \mathbb{P}\left(O K_{i}\right), \mathbb{P}\left(K O_{i}\right)$ & Successful and unsuccessful transmission through link $i$, and associated probabilities \\
\hline $\mathbb{P}($ all $K O), \mathbb{P}($ any $O K)$ & Probability of unsuccessful (successful) transmission through all links (at least one link) \\
\hline$K_{i, j}$ & Number of blocking elements that effectively block link $i$ or link $j$ \\
\hline$K_{\mathcal{A}}$ & Number of blocking elements that effectively block at least one of the links in the set $\mathcal{A}$ \\
\hline$|\mathcal{A}|$ & Cardinality of the set $\mathcal{A}$ (i.e., number of elements) \\
\hline$S_{i_{l w h \theta}}$ & Region containing centers of objects (with parameters $l, w, h, \theta$ ) blocking link $i$ \\
\hline$\left(x_{B}, y_{B}\right),\left(x_{U}, y_{U}\right),\left(x_{n}, y_{n}\right)$ & Position of BS, position of user, position of $n$th relay station (RS) \\
\hline$d, \phi, f_{D}(d), f_{\Phi}(\phi)$ & Distance to the center of the cell and azimuth of the user, and their corresponding pdf's \\
\hline$r, h_{R}, \psi_{n}$ & Distance to the center of the cell, height, and azimuth of $n$th RS \\
\hline$B U, B R_{n}, R_{n} U$ & BS-UE link, BS- $n$th RS link, $n$th RS-UE link \\
\hline$\tilde{S}_{B U}, \tilde{S}_{B R}, \tilde{S}_{R_{n} U}$ & $\begin{array}{l}\text { Sets of values }(d),\left(r, h_{R}\right) \text { and }\left(d, \phi, r, h_{R}\right) \text { associated to links } B U, B R_{n} \text {, and } R_{n} U \text {, } \\
\text { respectively, for which the received power is greater than the sensitivity }\end{array}$ \\
\hline$P_{T_{B}}, P_{T_{R}}, P_{R_{R}}, P_{R_{U}}$ & BS, RS transmissions powers, and RS, UE reception powers \\
\hline$G_{B}, G_{R}, G_{U}, S_{R}, S_{U}$ & BS, RS, UE antenna gains, and RS, UE sensitivities \\
\hline$R$ & Radius of the cell \\
\hline
\end{tabular}

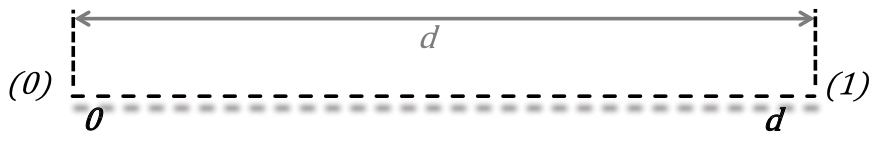

Fig. 1. Single-link with one transmitter and one receiver.

\section{Single-Link COMMUNiCATION}

In this section, we consider a single-link with one transmitter and one receiver, denoted as $(0)$ and (1) in Fig. 1, respectively, being $d$ the distance between them. As commented previously, in mmWave bands, a successful transmission requires LOS between (0) and (1), that is, no object blocking the segment connecting both nodes. We assume that the positions of the potentially blocking elements are random and follow a PPP and model the shapes and the sizes of these blocking elements as r.v.'s through random shape theory [14].

\section{A. Probability of Blockage}

Modeling the positions of the blocking elements as a PPP implies that:

- the number of potentially blocking objects in a given region is a Poisson r.v. (check [11]-[13], [23] for some references),
- the number of elements blocking a given concrete link (such as the one represented in Fig. 1), denoted by $K$, is a Poisson r.v. with parameter $\mathbb{E}[K]$ [24].

We compute the probability of having blockage following similarly to [8] and [9]. If we denote the probability of not having successful transmission due to blocking by $\mathbb{P}(K O)$, we have:

$$
\mathbb{P}(K O)=1-\mathbb{P}(K=0)=1-e^{-\mathbb{E}[K]} .
$$

Accordingly, the problem results in obtaining an analytic expression for $\mathbb{E}[K]$. We will present this for four different models of blocking elements detailed in subsection II-B (line and rectangular bases, with and without heights). The formulations presented in our paper in subsections II-B.1, II-B.2, II-B.3, and II-B.4 unify the methodologies and notations used in the previous papers [8]-[10]. This unification will allow us to extend this analysis to the multiple-link case and the relay-based scenario in Sections III and IV in this paper, also considering the four models.

\section{B. Modeling of the Blocking Elements Based on Random Shape Theory}

1) Line Segments Model: In this model, blocking elements are line segments of random lengths $L$ and orientations $\Theta$ 


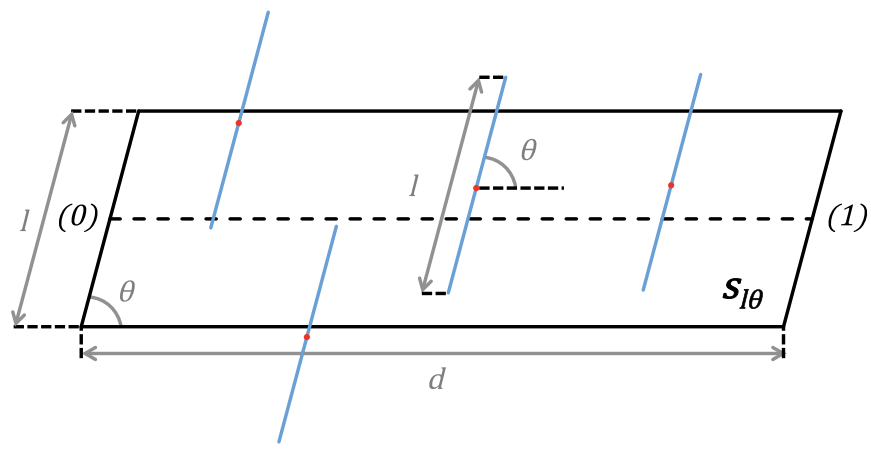

Fig. 2. Parallelogram $S_{l \theta}$ corresponding to the line segments model.

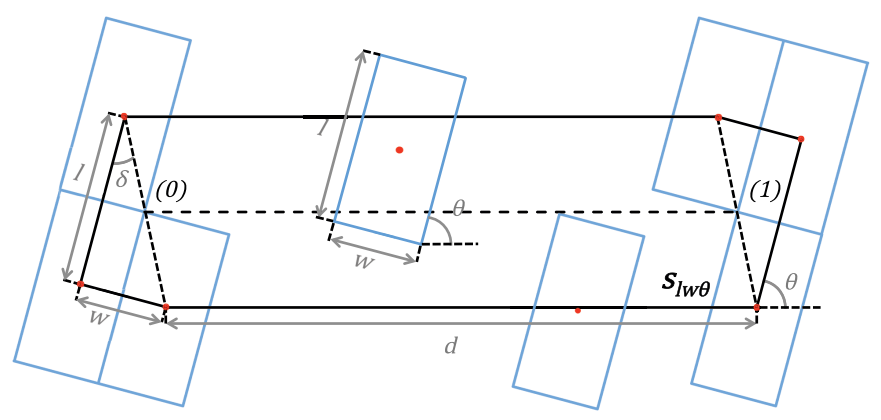

Fig. 3. Polygon $S_{l w \theta}$ corresponding to the rectangle model.

drawn from the pdf's $f_{L}(l)$ and $f_{\Theta}(\theta)$. Accordingly, the spatial density of blocking elements with lengths and orientations in the differential intervals $[l, l+d l]$ and $[\theta, \theta+d \theta]$, respectively, is given by $\lambda_{l \theta}=\lambda f_{L}(l) d l f_{\Theta}(\theta) d \theta$. Line segments of a given length $l$ and an orientation $\theta$ effectively block the link connecting nodes (0) and (1) of length $d$ if, and only if, their centers fall within the parallelogram $S_{l \theta}$ shown in Fig. 2 (see also Fig. 2 in [8]). In this case, $A_{S_{l \theta}}=|l d \sin \theta|$. Additionally, by assuming that the blocking elements can have any orientation with equal probability, ${ }^{1}$ that is, $f_{\Theta}(\theta)=\frac{1}{\pi}$ with $\Theta \sim \mathcal{U}[0, \pi]$, it is possible to write directly the modulus as $|l d \sin \theta|=l d \sin \theta$. Being $K_{l \theta}$ the number of line segments with length in $[l, l+d l]$ and orientation in $[\theta, \theta+d \theta]$ blocking the link, in [8] it is shown that $K_{l \theta} \sim \mathcal{P}\left(\bar{K}_{l \theta}\right)$, with mean value $\bar{K}_{l \theta}=\lambda_{l \theta} A_{S_{l \theta}}$. Taking everything into account, the total number of elements $K$ blocking the transmission is also a Poisson r.v. resulting from the aggregation of all the possible lengths and orientations with mean value

$$
\mathbb{E}[K]=\int_{l} \int_{\theta} \mathbb{E}\left[K_{l \theta}\right]=\beta d,
$$

where $\beta=\frac{2 \lambda \mathbb{E}[L]}{\pi}$.

2) Rectangles Model: In this model, each blocking element is a rectangle of length $l$, width $w$, and orientation $\theta$ drawn from the r.v.'s $L, W$, and $\Theta$, respectively. Accordingly, we denote by $S_{l w \theta}$ the geometric locus composed of the centers of all the possible blocking elements. For example, we have the polygon shown in Fig. 3 (see also Fig. 1 in [9]). In [9], it is shown that the number of blocking elements with

\footnotetext{
${ }^{1}$ From now on, we will make this assumption for the sake of simplicity, unless stated otherwise.
}

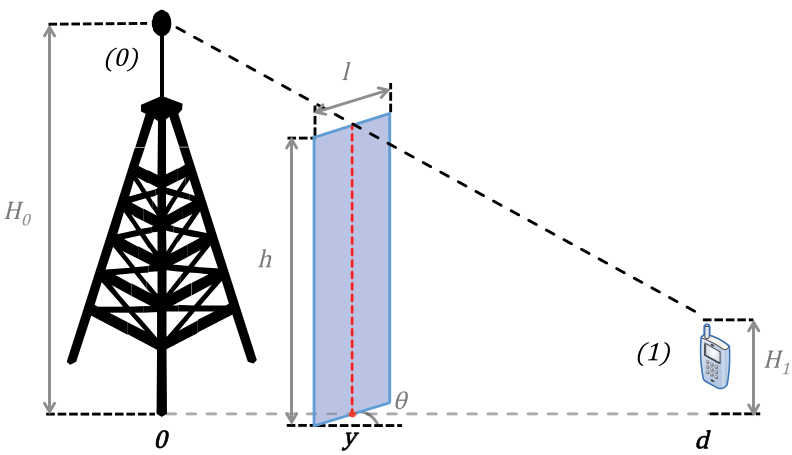

Fig. 4. Height effect on the line segments based model.

lengths, widths, and orientations in the differential intervals $[l, l+d l],[w, w+d w]$, and $[\theta, \theta+d \theta]$, respectively, denoted by $K_{l w \theta}$, is a Poisson r.v. with mean value given by $\bar{K}_{l w \theta}=$ $\lambda_{l w \theta} A_{S_{l w \theta}}$, where $\lambda_{l w \theta}=\lambda f_{L}(l) d l f_{W}(w) d w f_{\Theta}(\theta) d \theta$ and $A_{S_{l w \theta}}=d(l \sin \theta+w|\cos \theta|)+w l$.

Therefore, considering all the possible lengths, widths and orientations, in [9] it is shown that $K \sim \mathcal{P}(\beta d+p)$ with $\beta=\frac{2 \lambda(\mathbb{E}[L]+\mathbb{E}[W])}{\pi}$ and $p=\lambda \mathbb{E}[L] \mathbb{E}[W]$.

3) Line Segments with Height Model: The next step is to incorporate height in the line segments model, which means that the base of the blocking element is a line of length $l$ and orientation $\theta$, as previously, but a height $h$ is considered as well, obtaining a vertical rectangle, as illustrated in Fig. 4 (see also Fig. 2 in [9]). The values of $l, \theta$ and $h$ are drawn from the r.v.'s $L, \Theta$, and $H$. In the next figures (Fig. 4, 5 , and 6), the extreme points (0) and (1) of the link under analysis are considered to have also a certain height. For this reason, and to give an example, we have placed a BS and a mobile phone in (0) and in (1) with heights $H_{0}$ and $H_{1}$, respectively. This will help us in the understanding of the effect produced by the height of the blocking elements, addressed in this subsection II-B.3 and also in subsection II-B.4.

Following Fig. 4, the line segments placed at distance $y$ from (0) that effectively block the link are those with a height $h$ higher than the height of the link w.r.t. the ground at that point $y$.

As before, let $K$ denote the number of blocking elements that effectively block the considered link. $K$ follows a Poisson distribution whose mean is obtained as follows:

$$
\mathbb{E}[K]=\int_{l} \int_{h} \int_{\theta} \mathbb{E}\left[K_{l h \theta}\right]=\int_{l} \int_{h} \int_{\theta} \lambda_{l h \theta} A_{S_{l h \theta}},
$$

where $K_{l h \theta}$ is the number of elements blocking the link with lengths, heights, and orientations in the differential intervals $[l, l+d l],[h, h+d h]$, and $[\theta, \theta+d \theta]$, respectively. $K_{l h \theta}$ follows a Poisson distribution with mean value $\bar{K}_{l h \theta}=\lambda_{l h \theta} A_{S_{l h \theta}}$, where $\lambda_{l h \theta}=\lambda f_{L}(l) d l f_{H}(h) d h f_{\Theta}(\theta) d \theta$. What differs now from the previous cases is that the expression of $A_{S \ln \theta}$ changes depending on the considered $h$, as illustrated in Fig. 5. That figure shows the geometric locus $S_{\ln \theta}$ of the centers of the elements with length $l$, height $h$, and orientation $\theta$ blocking the link. Even though this region is a parallelogram, as in the line segments model (subsection II-B.1), there is a difference between these models: now, the length of the base of the parallelogram is $d-y$ instead of $d$ (which is the length for the 


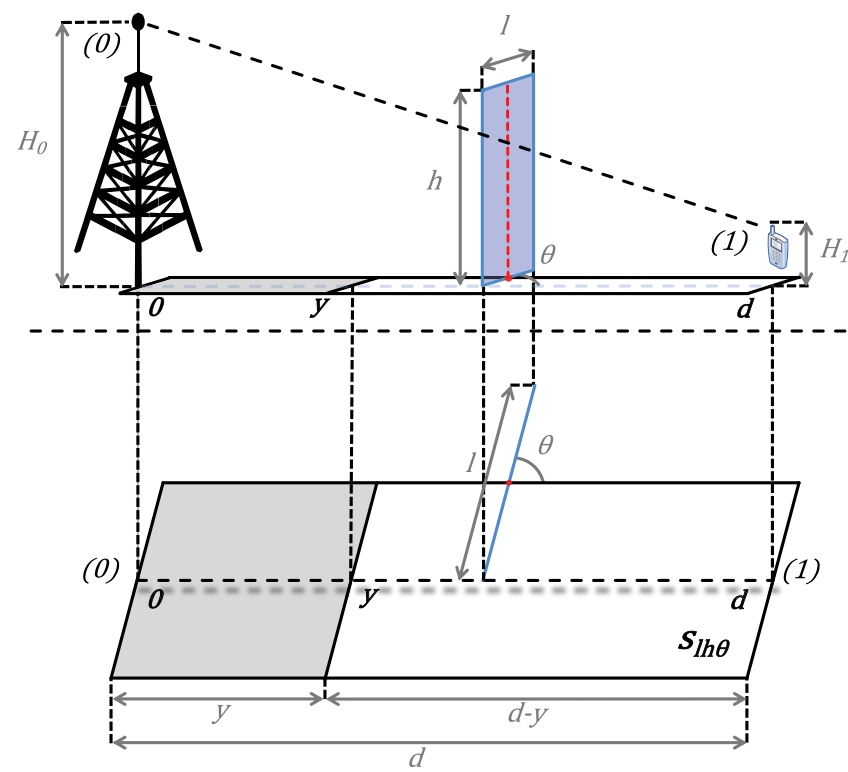

Fig. 5. Front view (above) and top view (below) of the blocking region $S_{l h \theta}$ (parallelogram) corresponding to the line segments with height model. The figure shows how the size of the parallelogram $S_{l h \theta}$ becomes smaller due to the height of the blocking elements.

case of segments without height shown in subsection II-B.1 and Fig. 2), therefore, it is $y$ meters smaller. By geometrical reasoning, $y$ is calculated as:

$$
y=\frac{H_{0}-h}{H_{0}-H_{1}} d \Rightarrow d-y=\frac{h-H_{1}}{H_{0}-H_{1}} d .
$$

In other words, the shadowed area is the region of $S_{l \theta}$ that, because of taking height into account, does not belong to the subset $S_{l h \theta}$. Finally, we can compute $A_{S_{l h \theta}}$ as:

$$
A_{S_{l h \theta}}= \begin{cases}l d \sin \theta & \text { if } h>H_{0} \\ \left(\frac{h-H_{1}}{H_{0}-H_{1}}\right) l d \sin \theta & \text { if } H_{1} \leq h \leq H_{0} \\ 0 & \text { if } h<H_{1} .\end{cases}
$$

By replacing $\lambda_{\operatorname{lh} \theta}$ and (5) in (3), we obtain

$$
\begin{aligned}
\mathbb{E}[K] & =\int_{l} \int_{h} \int_{\theta} \mathbb{E}\left[K_{l h \theta}\right] \\
& =\beta d\left(\int_{H_{1}}^{H_{0}} \frac{h-H_{1}}{H_{0}-H_{1}} f_{H}(h) d h+\int_{H_{0}}^{\infty} f_{H}(h) d h\right) \\
& =\beta d\left(1-\frac{1}{H_{0}-H_{1}} \int_{H_{1}}^{H_{0}} F_{H}(h) d h\right) \\
& =\eta \beta d,
\end{aligned}
$$

where $\beta=\frac{2 \lambda \mathbb{E}[L]}{\pi}$ and $\eta=1-\frac{1}{H_{0}-H_{1}} \int_{H_{1}}^{H_{0}} F_{H}(h) d h$, and $F_{H}(h)$ is the cumulative density function (cdf) of the r.v. $H$.

It can be concluded that adding height to the line segments model of blocking elements turns into a scaling factor $\eta$ over the mean number of blocking elements obtained in the line segments model. This coincides with the result in Subsection III.B in [9], although a different procedure has been followed.

4) Rectangles with Height Model: With this model, we can characterize the effect of 3D blockage produced by rectangular buildings with height (also addressed in [10]), which is pretty

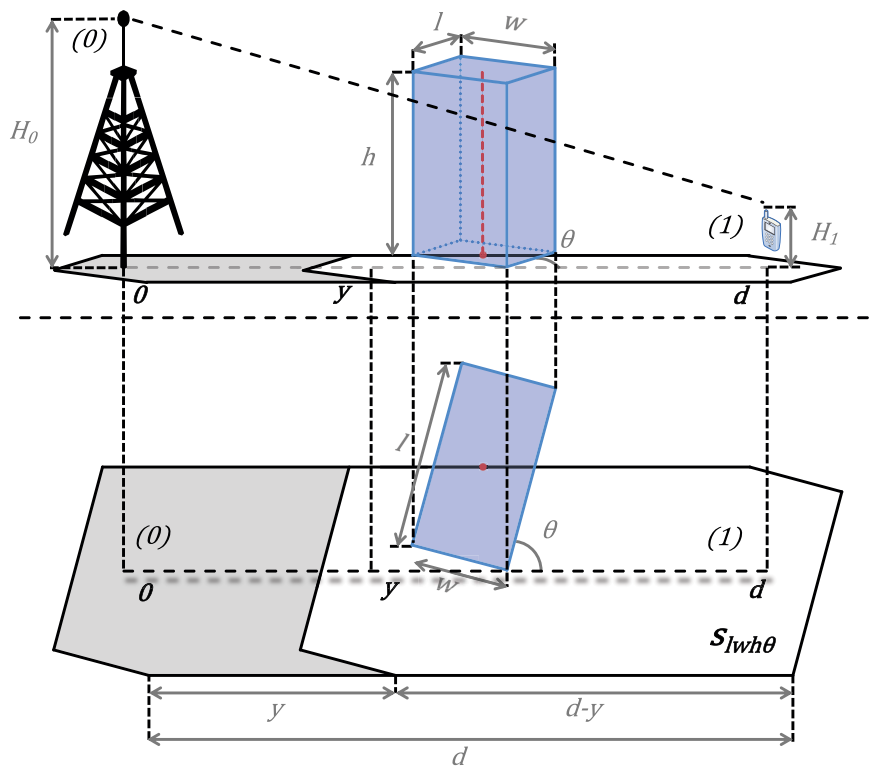

Fig. 6. Front view (above) and top view (below) of the blocking region $S_{l w h} \theta$ (polygon) corresponding to the rectangle with height model. The figure shows how the area of the polygon $S_{l w h \theta}$ becomes smaller due to the height of the blocking elements.

close to the real scenario that should be faced in cities. For each building, it is assumed that the dimensions and orientation $l, w, h$, and $\theta$ are drawn from the r.v.'s $L, W, H$, and $\Theta$, respectively.

As shown in Fig. 6, when incorporating height into the rectangle model, the effect is the same as in the line segments model: depending on the height of the blockage, the region $S_{l w h \theta}$ that contains the centers of all the blocking elements with length $l$, width $w$, height $h$, and orientation $\theta$ gets smaller when compared to Fig. 3 in subsection II-B.2, while the shape remains the same.

$K_{\text {lwh } \theta}$ is the number of elements blocking the link with lengths, widths, heights, and orientations in the differential intervals $[l, l+d l],[w, w+d w],[h, h+d h]$, and $[\theta, \theta+$ $d \theta$ ], respectively. It follows a Poisson distribution with mean value given by $\bar{K}_{l w h \theta}=\lambda_{l w h \theta} A_{S_{l w h \theta}}$, where $\lambda_{l w h \theta}=$ $\lambda f_{L}(l) d l f_{W}(w) d w f_{H}(h) d h f_{\Theta}(\theta) d \theta$ and

$$
A_{S_{l w h \theta}}=\left\{\begin{aligned}
d(l \sin \theta+w|\cos \theta|)+ & w l \\
& \text { if } h>H_{0} \\
\left(\frac{h-H_{1}}{H_{0}-H_{1}}\right) d(l \sin \theta+w \mid & \cos \theta \mid)+w l \\
0 & \text { if } H_{1} \leq h \leq H_{0}
\end{aligned}\right.
$$

The mean value of $K$, which is the total number of elements effectively blocking the transmission through the considered link that follows a Poisson distribution, is:

$$
\begin{aligned}
\mathbb{E}[K]= & \int_{l} \int_{w} \int_{h} \int_{\theta} \mathbb{E}\left[K_{l w h \theta}\right] \\
= & \beta d\left(\int_{H_{1}}^{H_{0}} \frac{h-H_{1}}{H_{0}-H_{1}} f_{H}(h) d h+\int_{H_{0}}^{\infty} f_{H}(h) d h\right) \\
& +p \int_{H_{1}}^{\infty} f_{H}(h) d h
\end{aligned}
$$




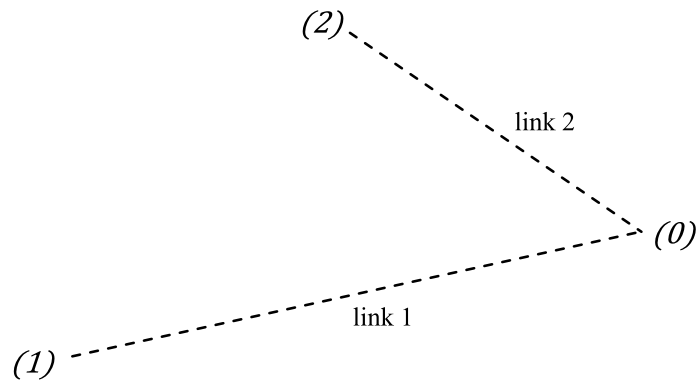

(a) 2 links.

Fig. 7. Multiple-link communication scenario.

$$
\begin{aligned}
= & \beta d\left(1-\frac{1}{H_{0}-H_{1}} \int_{H_{1}}^{H_{0}} F_{H}(h) d h\right) \\
& +p\left(1-F_{H}\left(H_{1}\right)\right) \\
= & \eta \beta d+\mu p
\end{aligned}
$$

with $\beta=\frac{2 \lambda(\mathbb{E}[L]+\mathbb{E}[W])}{\pi}, p=\lambda \mathbb{E}[L] \mathbb{E}[W], \eta=1-$ $\frac{1}{H_{0}-H_{1}} \int_{H_{1}}^{H_{0}} F_{H}(h) d h$, and $\mu=1-F_{H}\left(H_{1}\right)$. Note that the previous expression is more accurate than eq. (4) derived in [17]. In [17], it is considered that a 3D building does not block the link if the height at the center of the building does not block the vision, whereas in expression (8) it is considered the fact that LOS requires additionally that the faces of the 3D building do not block the vision.

Particularizing, if we assume $H \sim \mathcal{U}\left[0, H_{\max }\right]$, the parameters $\eta$ and $\mu$ can be easily derived, leading us to the expressions (9) and (10):

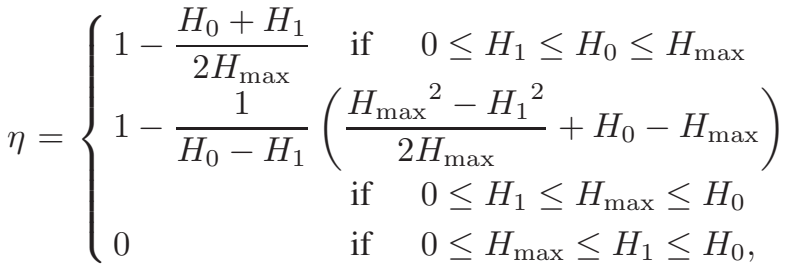

$$
\begin{aligned}
& \mu= \begin{cases}\frac{H_{\max }-H_{1}}{H_{\max }} & \text { if } 0 \leq H_{1} \leq H_{\max } \\
0 & \text { if } 0 \leq H_{\max } \leq H_{1}\end{cases}
\end{aligned}
$$

It is important to emphasize that considering generic pdf's for the lengths, widths, orientations, and heights of the blocking elements allows us to consider, as particular cases, several typical situations. For example, in urban environments, buildings may have deterministic widths or orientations. In such cases, the corresponding r.v.'s would just be deterministic and, consequently, the corresponding integrations can be calculated in closed-form (remember that for a deterministic variable $x$ taking value $x_{0}$, the pdf is given by $\delta\left(x-x_{0}\right)$ and $\left.\int g(x) \delta\left(x-x_{0}\right) d x=g\left(x_{0}\right)\right)$.

As an illustrative example, let us assume that all buildings have the same given widths, lengths, heights, and orientations denoted by $w_{0}, l_{0}, h_{b}$ (with $H_{1}<h_{b}<H_{0}$ ), and $\theta_{0}=0$, respectively. Then, the following simple closed-form

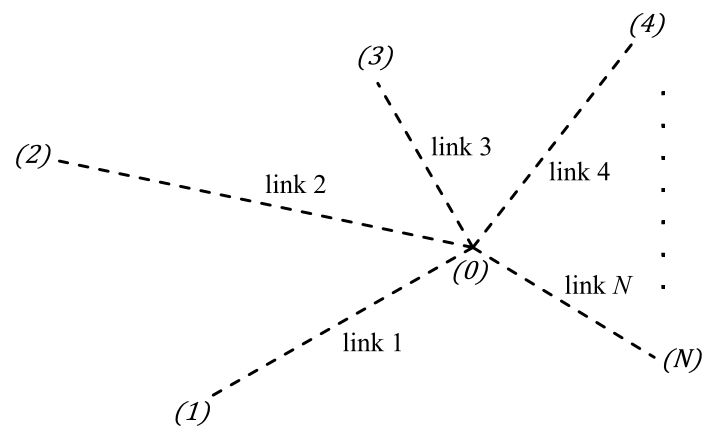

(b) $N$ links.

expression is obtained:

$$
\mathbb{E}[K]=\lambda w_{0}\left(\frac{h_{b}-H_{1}}{H_{0}-H_{1}} d+l_{0}\right) .
$$

Following the same procedure, other simplifications could be obtained by considering other cases of deterministic values for some of the parameters of the blocking objects.

\section{Multiple-Link Communication}

In the previous section, we have seen several ways of characterizing blocking in isolated links. Particularly, we have gone through four different models for the blocking elements and reached a general one consisting of the rectangles with height, which is the most realistic model for buildings in urban scenarios. This section generalizes the previous one by considering multiple links. See an example for 2 and $N$ links in Fig. 7(a) and 7(b), respectively.

Let $K_{i}$ and $K_{j}$ denote the number of blocking elements that effectively block the links $i$ and $j$, respectively, while $K_{i, j}$ is the number of blocking elements that obstruct link $i$ or link $j$. In general, when $\mathcal{A}$ is a set of links, $K_{\mathcal{A}}$ denotes the number of blocking elements that effectively block at least one of the links in that set.

In the following, $\mathbb{P}\left(O K_{i}\right)$ and $\mathbb{P}\left(K O_{i}\right)$ denote the probabilities of having and not having successful transmission through link $i$, respectively. On the other hand, $\mathbb{P}($ any $O K)$ is the probability of having successful transmission through, at least, one of the $N$ links, while $\mathbb{P}(\operatorname{all} K O)$ is the probability of not having successful transmission through any link.

The simplistic assumption that the blockings on each link are independent might not always hold. For instance, if two links are close in terms of the azimuth from a node's perspective, the probability that an element blockages both links simultaneously is high. In other words, the blockages that suffer two different distant UEs can have a non-zero correlation since that correlation between links depends both on the distance and the similarity of the angles under which each user observes the transmitter. Additionally, the hypothesis of independence does not hold when the lengths of links are small in terms of the blocking elements' length. This will be evaluated in the simulations section.

This section generalizes previous works such as [8]-[10] that only addressed single-link communications or did not 
consider correlation among links, and [6], [15] that took into account the correlation for the case of blockages modeled as segments without height. The derivations presented in this section are valid for the most general case of rectangles with height. In order to obtain the corresponding expressions, we will first consider only two links. Then, we will analyze the case of 3 links and, finally, generalize the expression to $N$ links.

\section{A. Two Links}

The calculation of $\mathbb{P}($ all $K O)$ and $\mathbb{P}($ any $O K)$ can be expressed as follows:

$$
\begin{aligned}
\mathbb{P}(\operatorname{all} K O) & =1-\mathbb{P}(\text { any } O K)=1-\mathbb{P}\left(O K_{1} \vee O K_{2}\right) \\
& =1-\mathbb{P}\left(O K_{1}\right)-\mathbb{P}\left(O K_{2}\right)+\mathbb{P}\left(O K_{1} \wedge O K_{2}\right) .
\end{aligned}
$$

While we have that $\mathbb{P}\left(O K_{1}\right)=e^{-\mathbb{E}\left[K_{1}\right]}$ and $\mathbb{P}\left(O K_{2}\right)=$ $e^{-\mathbb{E}\left[K_{2}\right]}, \mathbb{P}\left(O K_{1} \wedge O K_{2}\right)$ remains unknown. This term is the probability that there are not blockages neither in link 1 nor in link 2. To be general, we take the rectangles with height model of blocking elements. Let us first consider a specific length $l$, width $w$, height $h$, and orientation $\theta$ of the blocking elements. Accordingly, both links will be in LOS when no centers of the blocking elements fall within $S_{1_{l w h \theta}} \cup S_{2_{l w h \theta}}$. Generalizing the result to any length, width, height, and orientation and being $K_{1,2}$ the number of blockages that effectively block at least one of the 2 links, we can state that $\mathbb{P}\left(O K_{1} \wedge O K_{2}\right)=$ $\mathbb{P}\left(K_{1,2}=0\right)=e^{-\mathbb{E}\left[K_{1,2}\right]}$. Replacing it in (11), we obtain:

$$
\begin{aligned}
\mathbb{P}(\operatorname{all} K O) & =1-\mathbb{P}(\operatorname{any} O K)=1-e^{-\mathbb{E}\left[K_{1}\right]} \\
& -e^{-\mathbb{E}\left[K_{2}\right]}+e^{-\mathbb{E}\left[K_{1,2}\right]} .
\end{aligned}
$$

To make a quick comparison, we now present the probability of blockage assuming that blockages in every link are independent (an assumption that, in general, is not true):

$$
\begin{aligned}
\mathbb{P}(\operatorname{all} K O) & =\mathbb{P}\left(K O_{1}\right) \mathbb{P}\left(K O_{2}\right) \\
& =\left(1-e^{-\mathbb{E}\left[K_{1}\right]}\right)\left(1-e^{-\mathbb{E}\left[K_{2}\right]}\right) \\
& =1-e^{-\mathbb{E}\left[K_{1}\right]}-e^{-\mathbb{E}\left[K_{2}\right]}+e^{-\left(\mathbb{E}\left[K_{1}\right]+\mathbb{E}\left[K_{2}\right]\right)} .
\end{aligned}
$$

The difference is that in (12) we have the term $e^{-\mathbb{E}\left[K_{1,2}\right]}$ while, if we assume independence, we have $e^{-\left(\mathbb{E}\left[K_{1}\right]+\mathbb{E}\left[K_{2}\right]\right)}$ instead. For generalization purposes, considering the rectangles with height model of blocking elements, these expectations are expressed as follows:

$$
\left\{\begin{aligned}
\mathbb{E}\left[K_{1}\right]+\mathbb{E}\left[K_{2}\right] & =\int_{l} \int_{w} \int_{h} \int_{\theta} \lambda_{l w h \theta}\left(A_{S_{1_{l w h \theta}}}+A_{S_{2_{l w h \theta}}}\right) \\
\mathbb{E}\left[K_{1,2}\right] & =\int_{l} \int_{w} \int_{h} \int_{\theta} \lambda_{l w h \theta} A_{S_{1_{l w h \theta}} \cup S_{2_{l w h \theta}} .}
\end{aligned}\right.
$$

Therefore, we can see the difference between both expressions. When taking the statistical dependence between the blocking of the two links into consideration, it is assumed that both geometric loci may have a region in common, whose area is taken into account just once. In contrast to previous

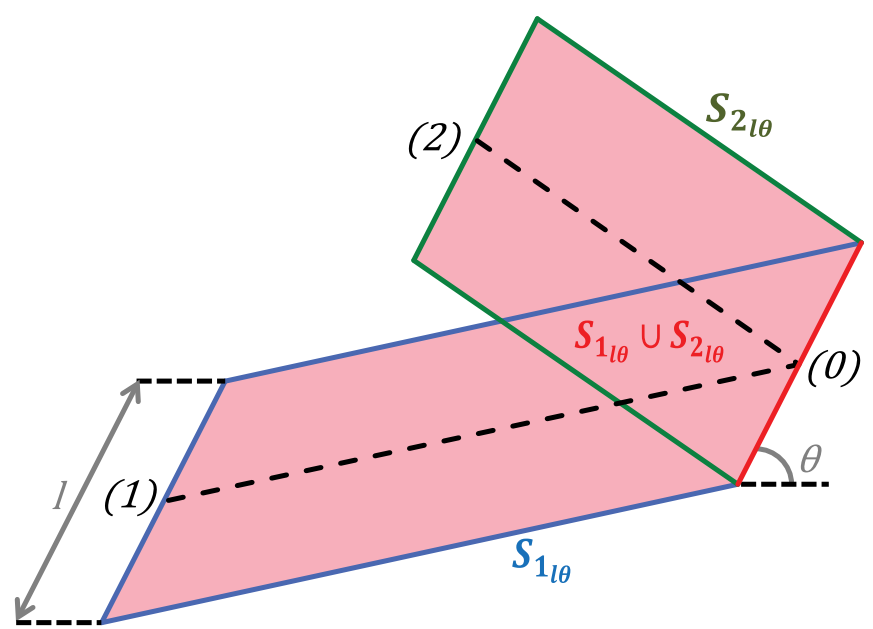

Fig. 8. Geometric loci associated to links 1 and $2, S_{1_{l \theta}}$ and $S_{2_{l \theta}}$, respectively, and the union, $S_{1_{l \theta}} \cup S_{2_{l \theta}}$, for the line segments model of blocking elements with length $l$ and orientation $\theta$.

assumption, what is done when considering independence between the blockages is to sum the area of this common region twice, which is incorrect. This effect is shown in Fig. 8. This makes that the probability of blockage when we consider the correlation of the blockings between the links is higher than in the other case.

Finally, if blockages in each link are independent, which happens whenever $A_{S_{l_{l w h}} \cup S_{2_{l w h \theta}}}=A_{S_{l_{l w h \theta}}}+$ $A_{S_{2_{l w h \theta}}} \forall l, w, h, \theta$ (that is, when the blocking regions do not overlap), our expression can also be applied (as stated in [15] as well). When this is not the case, the assumption of independence between the blockings of both links leads to inaccurate results, as will be shown in the simulations section.

\section{B. $N$ Links}

To generalize the expression above to the case of $N$ links, we first obtain the expression for three links. As we have done in the previous section, we are interested in the probability of blockage. Since now we are considering three links, we have:

$$
\begin{aligned}
\mathbb{P}(\operatorname{all} K O)= & 1-\mathbb{P}(\text { any } O K)=1-\mathbb{P}\left(O K_{1} \vee O K_{2} \vee O K_{3}\right) \\
= & 1-e^{-\mathbb{E}\left[K_{1}\right]}-e^{-\mathbb{E}\left[K_{2}\right]}-e^{-\mathbb{E}\left[K_{3}\right]}+e^{-\mathbb{E}\left[K_{1,2}\right]} \\
& +e^{-\mathbb{E}\left[K_{1,3}\right]}+e^{-\mathbb{E}\left[K_{2,3}\right]}-e^{-\mathbb{E}\left[K_{1,2,3}\right]}
\end{aligned}
$$

At this point, we are ready to generalize the expression to the case of $N$ links [25]:

$$
\begin{aligned}
\mathbb{P}(\text { all } K O)= & 1-\mathbb{P}(\text { any } O K)=1-\mathbb{P}\left(\bigvee_{n=1}^{N} O K_{n}\right) \\
= & 1-\sum_{n=1}^{N} \mathbb{P}\left(O K_{n}\right)+\sum_{n<m} \mathbb{P}\left(O K_{n} \wedge O K_{m}\right) \\
& -\sum_{n<m<l} \mathbb{P}\left(O K_{n} \wedge O K_{m} \wedge O K_{l}\right) \\
& +\cdots+(-1)^{N} \mathbb{P}\left(\bigwedge_{n=1}^{N} O K_{n}\right) .
\end{aligned}
$$


This could be written on closed form as follows (see [25]):

$$
\begin{aligned}
\mathbb{P}(\text { all } K O) & =1-\mathbb{P}\left(\bigvee_{n=1}^{N} O K_{n}\right) \\
& =1-\sum_{k=1}^{N}\left((-1)^{k-1} \sum_{\substack{\mathcal{A} \subset\{1, \ldots, N\} \\
|\mathcal{A}|=k}} \mathbb{P}\left(\bigwedge_{n \in \mathcal{A}} O K_{n}\right)\right),
\end{aligned}
$$

where $\mathcal{A}$ with $|\mathcal{A}|=k$ is a subset of $\{1, \ldots, N\}$ of $k$ links.

As previously explained, obtaining $\mathbb{P}\left(\bigwedge_{n \in \mathcal{A}} O K_{n}\right)$ is as simple as considering the area formed by the union of all the blocking regions associated with the links that form the subset $\mathcal{A}$ in each term. In other words:

$$
\mathbb{P}\left(\bigwedge_{n \in \mathcal{A}} O K_{n}\right)=e^{-\mathbb{E}\left[K_{\mathcal{A}}\right]}
$$

where $K_{\mathcal{A}}$ is the number of blocking elements that block, at least, one of the links that form the subset $\mathcal{A}$. This turns the former expression into:

$$
\begin{aligned}
& \mathbb{P}(\operatorname{all} K O)=1-\mathbb{P}\left(\bigvee_{n=1}^{N} O K_{n}\right) \\
& =1-\sum_{k=1}^{N}\left((-1)^{k-1} \sum_{\substack{\mathcal{A} \subset\{1, \ldots, N\} \\
|\mathcal{A}|=k}} e^{-\mathbb{E}\left[K_{\mathcal{A}}\right]}\right) \text {, }
\end{aligned}
$$

with

$$
\mathbb{E}\left[K_{\mathcal{A}}\right]=\int_{l} \int_{w} \int_{h} \int_{\theta} \lambda_{l w h \theta} A \underset{n \in \mathcal{A}}{\cup} S_{n_{l w h \theta}},
$$

when assuming the most general model of blocking elements, which are the rectangles with height. The only thing left is to obtain $A \cup \bigcup_{n \in \mathcal{A}} S_{n_{l w h \theta}}$, which is a matter of geometry. It should be highlighted that these results can be applied to any model of blocking elements. For instance, if the line segments model was considered, then the terms to obtain would be $A \underset{n \in \mathcal{A}}{\cup} S_{n_{l \theta}}$ instead.

\section{Application to Relay-Based Communications}

In previous sections, we have characterized the effect of blockages in isolated links and in the case of having multiple links, with different models of blocking elements, and taking correlation into account. In this section, we will use the previous generic results for a concrete application, namely the optimum positioning of a set of relays in a mmWave cell. A set of previous works for relays can be found in [19], [26]-[28], and references therein.

For this purpose, in the first subsection, we describe the scenario and consider some issues related to sensitivity and power loss. Finally, we will derive the expression of the probability of unsuccessful communication to minimize it by adjusting the positions of the relays.
This section generalizes the previous work [19]. There, a scenario with several nodes located at given non-random concrete positions is considered. In that paper, it is assumed that the blocking elements are circles with a given non-random radius and without height. That work considers that, whenever the direct link from the BS to a given node is blocked, it connects to a neighboring node that takes the role of relaying. In our work, we consider blocking elements with random shapes and calculate the blocking probability in a cell where there are several relays. This probability of not being in coverage is averaged over the user's random position, which was not done before. This allows for a global figure of merit in terms of coverage for the whole system and not only for a particular user position, based on which the positions of the relays can be optimized. Also, our work considers in this section that the receiver has a given sensitivity, which implies that the transmission will fail if the link length is too high due to signal attenuation even in a LOS situation. These sensitivity limitations are not considered in [19].

\section{A. Scenario and Problem Definition}

We consider a cell of radius $R$ with the BS placed at the origin, that is, $\left(x_{B}, y_{B}\right)=(0,0)$. This cell has $N$ RSs with height $h_{R}$ indexed by $n=1, \ldots, N$. The goal is to minimize the average probability that a UE does not achieve a successful transmission through any of the available links $\mathbb{P}($ all $K O)$, taking power and sensitivity constraints into account. Since we assume that the blockings are uniformly distributed within the considered cell with a certain spatial density, due to the symmetry of the problem, it is deduced that the optimum positions of the relays must be equispaced in azimuth. Therefore, the $n_{t h}$ RS is placed at $\left(x_{n}, y_{n}\right)=\left(r \cos \psi_{n}, r \sin \psi_{n}\right)$, where $\psi_{n}=(n-1) \frac{2 \pi}{N}$ is its azimuth and $r$ is the distance of the relays to the BS. The position of a generic UE can be expressed as $\left(x_{U}, y_{U}\right)=(d \cos \phi, d \sin \phi)$, where $\phi$ is the azimuth of the $\mathrm{UE}$ and $d$ is its distance to the BS. We assume that users are randomly uniformly distributed within the cell (which means than $\phi$ and $d$ are taken from the r.v.'s $\Phi$ and $D$, respectively). An example of the deployment with three RSs can be found in Fig. 9 .

As far as distances is concerned, we have the following relations:

$\left\{\begin{array}{l}\left\|\left(x_{B}, y_{B}\right)-\left(x_{n}, y_{n}\right)\right\|=r \quad \forall n \\ \left\|\left(x_{n}, y_{n}\right)-\left(x_{U}, y_{U}\right)\right\|=\sqrt{d^{2}+r^{2}-2 d r \cos \left(\phi-\psi_{n}\right)} \\ \left\|\left(x_{B}, y_{B}\right)-\left(x_{U}, y_{U}\right)\right\|=d\end{array}\right.$

The analysis performed in this section focuses on two different types of cells:

- Sectorized cells: the cell is divided into $N$ sectors, which are served by a single RS each. A UE that is in the $n_{t h}$ sector can be connected to just the $n_{t h}$ RS or to the BS itself, but not to any other RS.

- Non-sectorized cells: each user can be connected to any RS of the cell or to the BS.

For the sake of simplicity, we consider the downlink transmission. A set of parameters related to power and propagation 


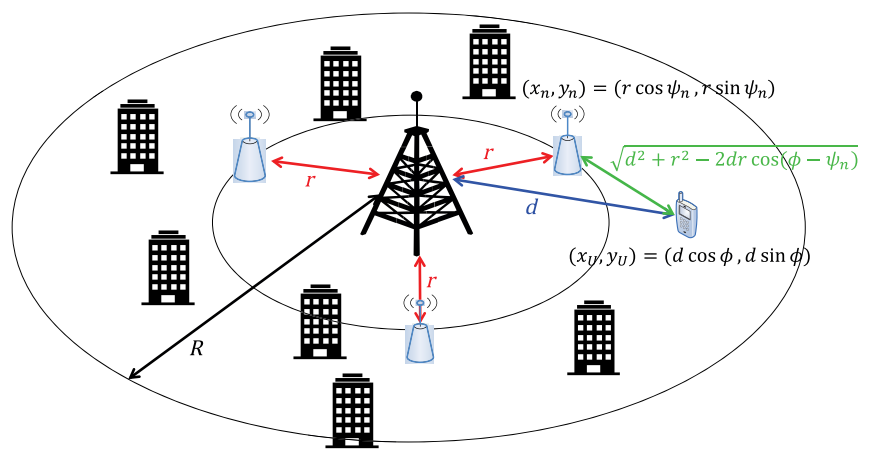

Fig. 9. Example of a deployment with 3 RSs.

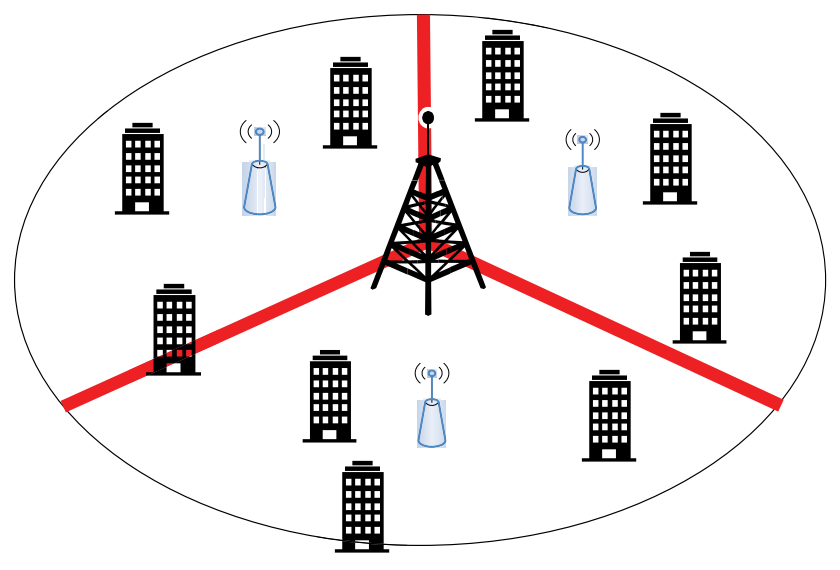

Fig. 10. Example of a deployment with 3 RSs in a sectorized cell.

are defined in Table I. The heights are only used when talking about models of blocking elements that incorporate height.

When considering that the received power must be greater than the sensitivity and taking propagation losses into account, we have a set of constraints that can be formulated through the following indicative functions, in which height is considered: $\mathbb{1}_{\tilde{S}_{B U}}(d), \mathbb{1}_{\tilde{S}_{B R}}\left(r, h_{R}\right)$, and $\mathbb{1}_{\tilde{S}_{R_{n} U}}\left(d, \phi, r, h_{R}\right) . \tilde{S}_{B U}$, $\tilde{S}_{B R}$, and $\tilde{S}_{R_{n} U}$ are the sets of values $(d),\left(r, h_{R}\right)$, and $\left(d, \phi, r, h_{R}\right)$ associated to links $B U, B R$, and $R_{n} U$, respectively, for which the sensitivity conditions are fulfilled. These additional constraints also affect the probability of having successful/unsuccessful transmission and will be incorporated into the computation of $\mathbb{P}($ all $K O)$ in what follows.

\section{B. Probability of Successful Transmission (Average Coverage Probability)}

The objective is to compute the position of the relays (i.e., distance $r$ to the BS) and their height to minimize the average probability of not having successful transmission, that is,

$$
\min _{r, h_{R}} \int_{0}^{R} \int_{\phi_{s}}^{\phi_{e}} \mathbb{P}(\operatorname{all} K O \mid D=d, \Phi=\phi) f_{D}(d) f_{\Phi}(\phi) d d d \phi,
$$

where $f_{D}(d)$ and $f_{\Phi}(\phi)$ are the pdf's of the distance and azimuth of a user located at a random position, which is detailed in what follows.
Note that this is the general expression and can have many versions. For instance, if we consider the line segments or the rectangles models of blocking elements, then heights will not be taken into account and the height of the RSs $h_{R}$ will not appear in the expressions.

On the other hand, the limits of the integral w.r.t. the azimuth $\phi$ and the pdf $f_{\Phi}(\phi)$ depend on whether the cell is sectorized or not, as explained above. Consequently, we focus only on the term $\mathbb{P}(\operatorname{all} K O \mid D=d, \Phi=\phi)$. Additionally, from now on, and for the sake of simplicity, we omit to write $\mid D=d, \Phi=\phi$, but it should not be forgotten that the expressions that follow are just for a specific position of the UE and the RS.

1) Sectorized Cells: As explained above, in sectorized cells, cells are divided into $N$ different sectors and the UE can be only connected to the RS of the sector where it is located or directly to the BS (see Fig. 10).

A UE is considered to be within the $n_{t h}$ sector whenever its azimuth is between $\psi_{n_{s}}=\psi_{n}-\frac{\pi}{N}$ and $\psi_{n_{e}}=\psi_{n}+\frac{\pi}{N}$, which are the angles that limit that region. With this in mind, in this case, the random position of a user in the $n_{t h}$ sector can be characterized by the following pdf's ${ }^{2}$ :

$$
\left\{\begin{array}{l}
f_{D}(d)=\frac{2 d}{R^{2}} \quad \text { where } \quad 0 \leq d \leq R \\
f_{\Phi}(\phi)=\frac{N}{2 \pi} \quad \text { where } \quad \psi_{n_{s}} \leq \phi \leq \psi_{n_{e}} .
\end{array}\right.
$$

By applying (19) and (20) to sectorized cells and incorporating the power limitations through the corresponding indicative functions, we have that the probability of not having successful transmission is:

$$
\begin{aligned}
\mathbb{P}(\operatorname{all} K O)= & -\mathbb{P}\left(O K_{B U}\right)-\mathbb{P}\left(O K_{B R_{n}} \wedge O K_{R_{n} U}\right) \\
& +\mathbb{P}\left(O K_{B U} \wedge O K_{B R_{n}} \wedge O K_{R_{n} U}\right) \\
= & 1-e^{-\mathbb{E}\left[K_{B U}\right]} \mathbb{1}_{\tilde{S}_{B U}}(d) \\
& -e^{-\mathbb{E}\left[K_{B R_{n}, R_{n} U}\right]_{\mathbb{1}} \tilde{S}_{B R}}\left(r, h_{R}\right) \mathbb{1}_{\tilde{S}_{R_{n} U}}\left(d, \phi, r, h_{R}\right) \\
& +e^{-\mathbb{E}\left[K_{B U, B R_{n}, R_{n} U}\right]} \mathbb{1}_{\tilde{S}_{B U}}(d) \\
& \mathbb{1}_{\tilde{S}_{B R}}\left(r, h_{R}\right) \mathbb{1}_{\tilde{S}_{R_{n} U}}\left(d, \phi, r, h_{R}\right) .
\end{aligned}
$$

The only thing left is to calculate the area of the union of such blocking regions for every possible length, width, height, and orientation of the blocking elements if, for instance, the rectangles with height model, which is the most general one, is considered. Fig. 11 shows an example with three parallelograms associated to the $B U, B R_{n}$, and $R_{n} U$ links when considering the line segments model of blocking elements with a specific length $l$ and orientation $\theta$.

In some practical cases, relays may be placed in a way such that no blockings between them and the BS will happen. In other words, the $B R_{n}$ link will be in LOS, that is

${ }^{2}$ Since we assume users uniformly distributed within the cell, a division of areas gives us that $\operatorname{Pr}(D \leq d)=\frac{\pi d^{2}}{\pi R^{2}}=\frac{d^{2}}{R^{2}}$. Therefore, we obtain, through derivation, $f_{D}(d)=\frac{2 d}{R^{2}}$. This pdf applies to both sectorized and non-sectorized cells.

On the other hand, $f_{\Phi}(\phi)$ is a uniform pdf whose margin depends on whether the cell is sectorized or not. 


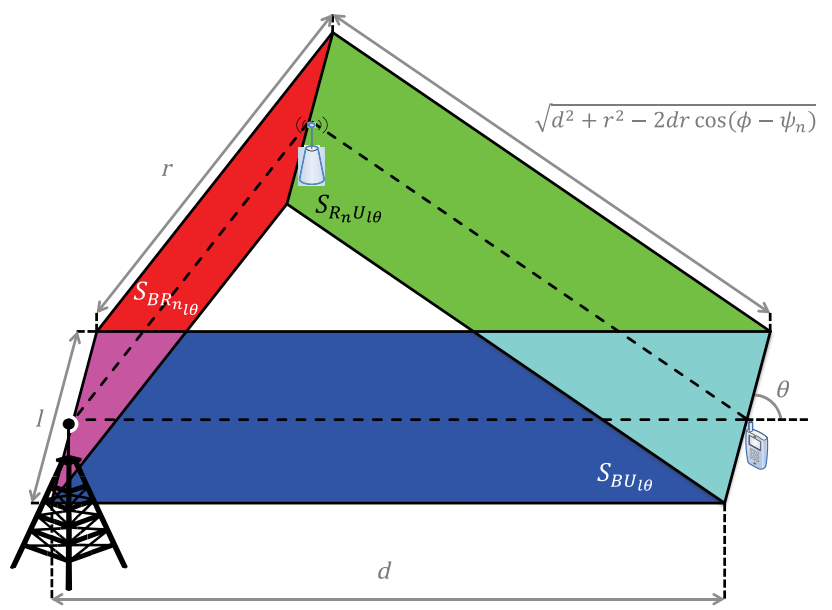

Fig. 11. Parallelograms of the three links in a sectorized cell, with line segments as blocking elements with length $l$ and orientation $\theta$.

$K_{B R_{n}}=0$. This makes that $K_{B R_{n_{l w h} \theta}}=0 \forall l, w, h, \theta$, which means that no centers of blockages fall within $S_{B R_{n_{l w h \theta}}}$ and, therefore, neither in $S_{B R_{n_{l w h \theta}}} \cap S_{R_{n} U_{l w h \theta}}$ nor in $S_{B R_{n}{ }_{l w h \theta}} \cap$ $S_{B U_{l w h \theta}}$. Consequently,

$$
\begin{aligned}
& \mathbb{P}(\operatorname{all} K O)=1-\mathbb{P}\left(O K_{B U}\right)-\mathbb{P}\left(O K_{B R_{n}} \wedge O K_{R_{n} U}\right) \\
& +\mathbb{P}\left(O K_{B U} \wedge O K_{B R_{n}} \wedge O K_{R_{n} U}\right) \\
& =1-e^{-\mathbb{E}\left[K_{B U} \mid K_{B R_{n}}=0\right]} \mathbb{1}_{\tilde{S}_{B U}}(d) \\
& -e^{-\mathbb{E}\left[K_{R_{n} U} \mid K_{B R_{n}}=0\right]} \mathbb{1}_{\tilde{S}_{B R}}\left(r, h_{R}\right) \\
& \mathbb{1}_{\tilde{S}_{R_{n} U}}\left(d, \phi, r, h_{R}\right) \\
& +e^{-\mathbb{E}\left[K_{B U, R_{n} U} \mid K_{B R_{n}}=0\right]} \mathbb{1}_{\tilde{S}_{B U}}(d) \\
& \mathbb{1}_{\tilde{S}_{B R}}\left(r, h_{R}\right) \mathbb{1}_{\tilde{S}_{R_{n} U}}\left(d, \phi, r, h_{R}\right),
\end{aligned}
$$

where

$$
\left\{\begin{aligned}
\mathbb{E} & {\left[K_{B U} \mid K_{B R_{n}}=0\right] } \\
& =\int_{l} \int_{w} \int_{h} \int_{\theta} \lambda_{l w h \theta} A_{S_{B U_{l w h \theta}} \backslash S_{B R_{n}}} \\
\mathbb{E} & {\left[K_{R_{n} U} \mid K_{B R_{n}}=0\right] } \\
& =\int_{l} \int_{w} \int_{h} \int_{\theta} \lambda_{l w h \theta} A_{S_{R_{n} U_{l w h \theta}} \backslash S_{B R_{n}}} \\
\mathbb{E} & {\left[K_{B U, R_{n} U} \mid K_{B R_{n}}=0\right] } \\
& =\int_{l} \int_{w} \int_{h} \int_{\theta} \lambda_{l w h \theta} A_{\left(S_{B U_{l w h \theta}} \cup S_{R_{n} U_{l w h \theta}}\right) \backslash S_{B R_{n w h \theta}}} .
\end{aligned}\right.
$$

2) Non-Sectorized Cells: If the cell is not sectorized, that is, if a user can be connected to any RS in the cell, the position of the user follows the following distributions ${ }^{2}$ :

$$
\left\{\begin{array}{l}
f_{D}(d)=\frac{2 d}{R^{2}} \quad \text { where } \quad 0 \leq d \leq R \\
f_{\Phi}(\phi)=\frac{1}{2 \pi} \quad \text { where } \quad 0 \leq \phi \leq 2 \pi .
\end{array}\right.
$$

Gathering everything together, the formulation of not having a successful transmission in the not sectorized case with $N$ relays is (in the following expressions, $n=N+1$ refers to the direct link between the BS and the UE):

$$
\begin{aligned}
\mathbb{P}(\operatorname{all} K O) & =1-\mathbb{P}\left(\bigvee_{n=1}^{N+1} O K_{n}\right) \\
= & 1-\sum_{k=1}^{N+1}\left((-1)^{k-1} \sum_{\substack{\mathcal{A} \subset\{1, \ldots, N+1\} \\
|\mathcal{A}|=k}} \mathbb{P}\left(\bigwedge_{n \in \mathcal{A}} O K_{n}\right)\right) \\
= & \sum_{k=1}^{N+1}\left((-1)^{k-1} \sum_{\substack{\mathcal{A} \subset\{1, \ldots, N+1\} \\
|\mathcal{A}|=k}}\left(e^{-\mathbb{E}\left[K_{\mathcal{A}}\right]} \prod_{n \in \mathcal{A}} \mathbb{1}_{\tilde{S}_{n}}\left(d, \phi, r, h_{R}\right)\right)\right),
\end{aligned}
$$

with

$$
\mathbb{1}_{\tilde{S}_{n}}\left(d, \phi, r, h_{R}\right)=\left\{\begin{array}{rll}
\mathbb{1}_{\tilde{S}_{B R}}\left(r, h_{R}\right) \mathbb{1}_{\tilde{S}_{R_{n} U}}\left(d, \phi, r, h_{R}\right) \\
& \text { if } & n \leq N \\
\mathbb{1}_{\tilde{S}_{B U}}(d) & \text { if } & n=N+1 .
\end{array}\right.
$$

If we consider the rectangle based model with height, we have:

$$
\begin{aligned}
\mathbb{E}\left[K_{\mathcal{A}}\right] & =\int_{l} \int_{w} \int_{h} \int_{\theta} \mathbb{E}\left[K_{\mathcal{A}_{l w h \theta}}\right] \\
& =\int_{l} \int_{w} \int_{h} \int_{\theta} \lambda_{l w h \theta} A \underset{n \in \mathcal{A}}{\cup} S_{n_{l w h}},
\end{aligned}
$$

where $\lambda_{l w h \theta}=\lambda f_{L}(l) d l f_{W}(w) d w f_{H}(h) d h f_{\Theta}(\theta) d \theta$ and

$$
S_{n_{l w h \theta}}= \begin{cases}S_{B R_{n_{l w h \theta}}} \cup S_{R_{n} U_{l w h \theta}} & \text { if } n \leq N \\ S_{B U_{l w h \theta}} & \text { if } n=N+1 .\end{cases}
$$

The assumption that all the $B R$ links are in LOS, that is, there are no blockages between the BS and the RS, can also be made here, which implies that

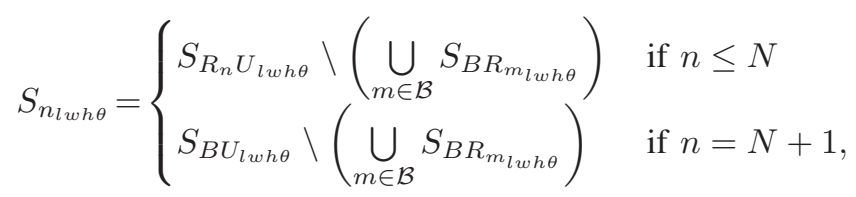

where $\mathcal{B}=\{1, \ldots N\}$.

\section{RESUlts}

To validate the analytic expressions derived in this work, we will compare them with some Monte Carlo numerical simulations in which blockages are thrown randomly within a cell following a uniform spatial PPP distribution. The model of blocking elements considered is the one of rectangles with height. 
TABLE II

Values of the Parameters Taken in the Simulations

\begin{tabular}{|c|c|c|}
\hline Parameter & Description & Value \\
\hline \hline$R$ & Cell radius & $300 \mathrm{~m}$ \\
\hline$H_{B}$ & BS height & $40 \mathrm{~m}$ \\
\hline$H_{U}$ & UE height & $1.5 \mathrm{~m}$ \\
\hline$L_{\max }, W_{\max }, H_{\max }$ & Maximum length, width, height of buildings & $30 \mathrm{~m}, 30 \mathrm{~m}, 30 \mathrm{~m}$ \\
\hline$\Theta_{\max }$ & Maximum orientation of buildings & $\pi \mathrm{rad}$ \\
\hline$P_{T_{B}}$ & BS transmission power & $25 \mathrm{dBm}$ \\
\hline$P_{T_{R}}$ & RS transmission power & $20 \mathrm{dBm}$ \\
\hline$S_{R}$ & RS sensitivity & $-90.2 \mathrm{dBm}$ \\
\hline$S_{U}$ & UE sensitivity & $-79.5 \mathrm{dBm}$ \\
\hline$G_{B}$ & BS antenna gain & $23 \mathrm{dBi}$ \\
\hline$G_{R}$ & RS antenna gain & $23 \mathrm{dBi}$ \\
\hline$G_{U}$ & UE antenna gain & $0 \mathrm{dBi}$ \\
\hline$f$ & Frequency & $28 \mathrm{GHz}$ \\
\hline$\alpha$ & Path-loss exponent & 2.3 \\
\hline
\end{tabular}

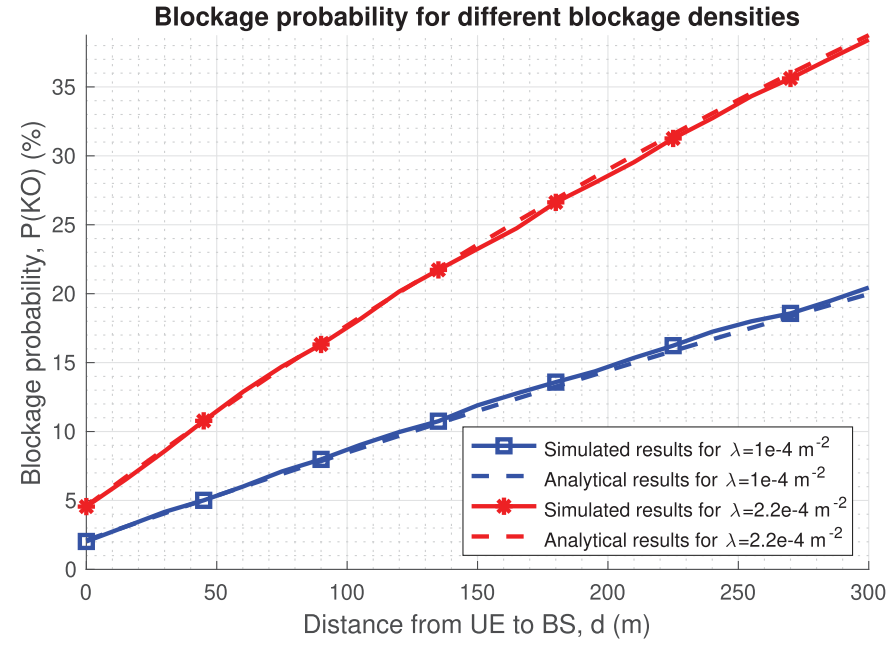

Fig. 12. Blockage probability vs distance of the UE to the BS for two possible blockage densities. No power budget limitations are considered.

\section{A. Single-Link}

In this subsection, we consider a single-link (i.e., no relays are deployed) without considering sensitivity, that is, without considering power limitations. The values of the parameters in this simulation are detailed in Table II.

First, we obtain the probability that a UE at a given distance $d$ from the BS (which is at the center of the cell) is blocked. This distance is evaluated from 0 to the radius $R$ of the cell. Following the expressions for the blockage probability in (1) and the one of the mean value of the number of blockages with the rectangles with height model in (8), we derive the formula to obtain the blockage probability analytically. In Fig. 12, we take blocking elements (that is, buildings) with their lengths, widths, heights, and orientations following uniform distributions: $L \sim \mathcal{U}\left[0, L_{\max }\right], W \sim \mathcal{U}\left[0, W_{\max }\right]$, $H \sim \mathcal{U}\left[0, H_{\max }\right]$, and $\Theta \sim \mathcal{U}\left[0, \Theta_{\max }\right]$ with maximum values shown in Table II. We analyze the blockage probability for the cases where the density of blockages $\lambda$ is $1 \cdot 10^{-4} \mathrm{~m}^{-2}$ and $2.2 \cdot 10^{-4} \mathrm{~m}^{-2}$.

We can see that the simulation results perfectly match the analytical results. The relation between the blockage probability, $P(K O)$, and the distance $d$ to the $\mathrm{BS}$ is almost linear. As expected, the denser the buildings are and the further from the BS the users are located, the more likely the UEs are blocked.

Note that, as already observed in [9], the blockage probability is not zero when the $2 \mathrm{D}$ distance (i.e., horizontal distance) between transmitter and receiver is zero. This is because there is a non-zero probability that the user is located within a building, while the BS is outdoor. In this case, the building will block the signal transmission between the BS and the indoor users.

Next, we want to see in detail the influence of the density of blockages on the overall blockage probability of the users within the cell. To obtain the expression of the mean blockage probability of all the cell, we take into account again expressions (1) and (8), which have allowed us to obtain the blockage probability at a given position. In this case we should take the mean value by considering that users are distributed uniformly throughout the cell following the same distributions that we had in (27). Then, the mean analytic probability of blockage is:

$$
\begin{aligned}
\overline{\mathbb{P}}(K O) & =\int_{0}^{2 \pi} \int_{0}^{R} \mathbb{P}(K O \mid D=d, \Phi=\phi) f_{D}(d) f_{\Phi}(\phi) d d d \phi \\
& =\int_{0}^{2 \pi} \int_{0}^{R}\left(1-e^{-\left(\eta_{B U} \beta d+\mu_{B U} p\right)}\right) d \frac{2}{R^{2}} \frac{1}{2 \pi} d d d \phi \\
& =1+\frac{2\left(\eta_{B U} \beta R-e^{\eta_{B U} \beta R}+1\right)}{\left(\eta_{B U} \beta R\right)^{2}} e^{-\left(\eta_{B U} \beta R+\mu_{B U} p\right)},
\end{aligned}
$$

where $\eta_{B U}$ and $\mu_{B U}$ are the $\eta$ and $\mu$ parameters particularized for the BU link. In Fig. 13, the values of all the parameters are the same as before except the maximum height $H_{\max }$ of the blocking elements. Now, in addition to the height of $30 \mathrm{~m}$ considered before, we also considered $40 \mathrm{~m}$ to gain more insights into the scenario. In Fig. 13 we can see the results, from which it can be concluded, as expected, that the higher and denser the buildings are, the more likely the users are going to be blocked. 


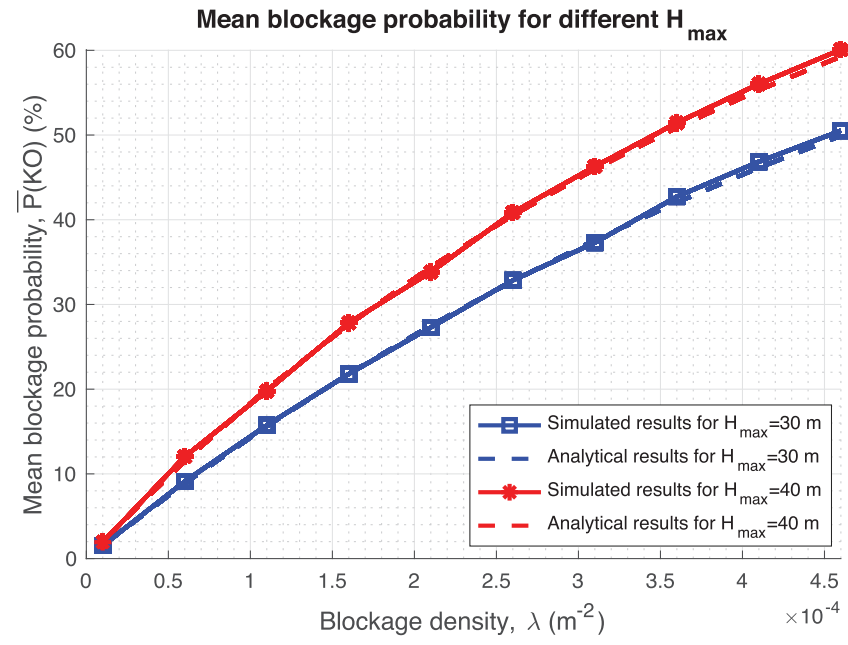

Fig. 13. Average average blockage probability depending on the blockage density and the maximum height of the buildings. No power budget limitations are considered.

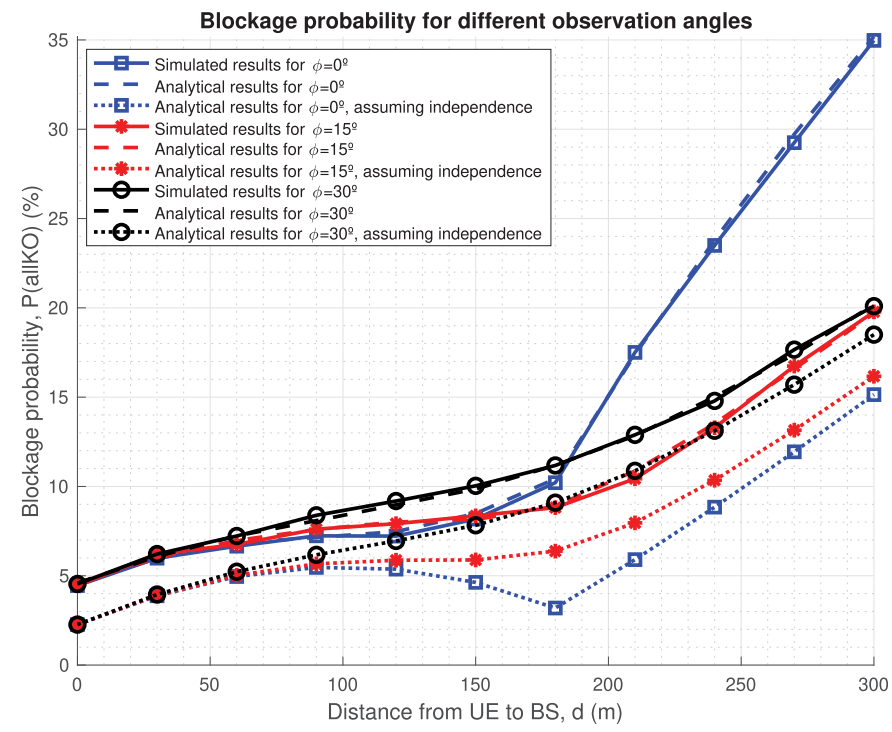

Fig. 14. Blockage probability at a given distance from the BS depending on the azimuth of observation. No power budget limitations are considered.

\section{B. Relay Deployment}

This subsection validates and analyzes the results for the case in which three RSs are deployed within the cell. Here we consider the sectorized case; that is, the user can only be connected to the BS directly or via the RS of the sector where it is located.

In the first case, shown in Fig. 14, we are interested in comparing the simulation with the analytic results. Relays are placed at a distance of $r=180 \mathrm{~m}$ from the BS and at a height of $h_{R}=20 \mathrm{~m}$. Both the length $L$ and width $W$ of the blockages are set to $15 \mathrm{~m}$, while the height and orientation again follow a uniform distribution such that $H \sim \mathcal{U}\left[0, H_{\max }\right]$ with $H_{\max }=30 \mathrm{~m}$ and $\Theta \sim \mathcal{U}\left[0, \Theta_{\max }\right]$ with $\Theta_{\max }=\pi \mathrm{rad}$, respectively. No power limitations are considered. Then, as we had in the single-link situation of Fig. 12, we evaluate the probability of blockage that a user experiences at different distances from the BS. Since we are placing RSs, it is important to take into account the azimuth at which the user is located, since we will obtain different results depending on whether the azimuth of the user is similar or not to the azimuth of the RS in the corresponding sector.

Specifically, we assume that the first of the 3 RSs is placed at $\psi_{1}=0^{\circ}$, whose sector lies between $\psi_{1_{s}}=-60^{\circ}$ and $\psi_{1_{e}}=60^{\circ}$. Due to symmetry among the three sectors, we only focus on this first sector and obtain the blockage probability as a function of the distance $d$ between the UE and the BS for three different azimuths of the user, namely $\phi=0^{\circ}, \phi=15^{\circ}$, and $\phi=30^{\circ}$. For each angle, we show the simulated results, the analytical results, and the analytical results assuming independence among the blocking elements of each link.

- UE at $\phi=0^{\circ}$ (blue lines with square markers): in this situation, the azimuths of the user and the RS are the same. We can see that if the user is located at a distance greater than $180 \mathrm{~m}$ from the center, there is a sharp increase in the blockage probability. This is because, in this case, we are not exploiting the diversity gain among the blocking elements of the different links. In other words: if the link between the BS and the RS is blocked, the communication via the RS will not be possible. Furthermore, since the UE's height is smaller than the RS's height, the BU link will be blocked. Consequently, the three links are highly correlated. Regarding the assumption that the blocking elements are independent in each link, we can see that the result is entirely different, leading us to more optimistic results. As commented, if the BR link is blocked, the BU link will be blocked, too. However, the independence assumption considers that this may not always be the case, which is not realistic. This is the reason why the independence assumption produces lower blockage probabilities.

- $U E$ at $\phi=15^{\circ}$ (red lines with asterisk markers): in this case, by moving azimuthally $15^{\circ}$ aside from $\psi_{1}$, we see that the results are much better than in the previous case. This is for the same reason as discussed before: in this situation, if the BR link is blocked, the BU link may not be blocked, and viceversa. Here we are exploiting the diversity of blockages, and we do not find the previous sharp increase in the blockage probability for $d=180 \mathrm{~m}$.

- UE at $\phi=30^{\circ}$ (black lines with circle markers): in this latter situation, the blockage probability from $d=180 \mathrm{~m}$ is lower than in the first one of $\phi=0^{\circ}$ because, again, it exploits the diversity gain among the blocking elements in the different links, but it is not lower than in the case of $\phi=15^{\circ}$. This is for an apparent reason: even though we have the effect of this diversity gain, at $\phi=30^{\circ}$ the UE is farther from the RS than in the $\phi=15^{\circ}$ case, which makes more likely to have more blocking elements in the RU link and, therefore, the blockage probability increases.

In conclusion, even though we have not considered power budget limitations, there is a trade-off between exploiting the diversity of the blocking elements among the different links and not being located very far from the RS to reduce the 


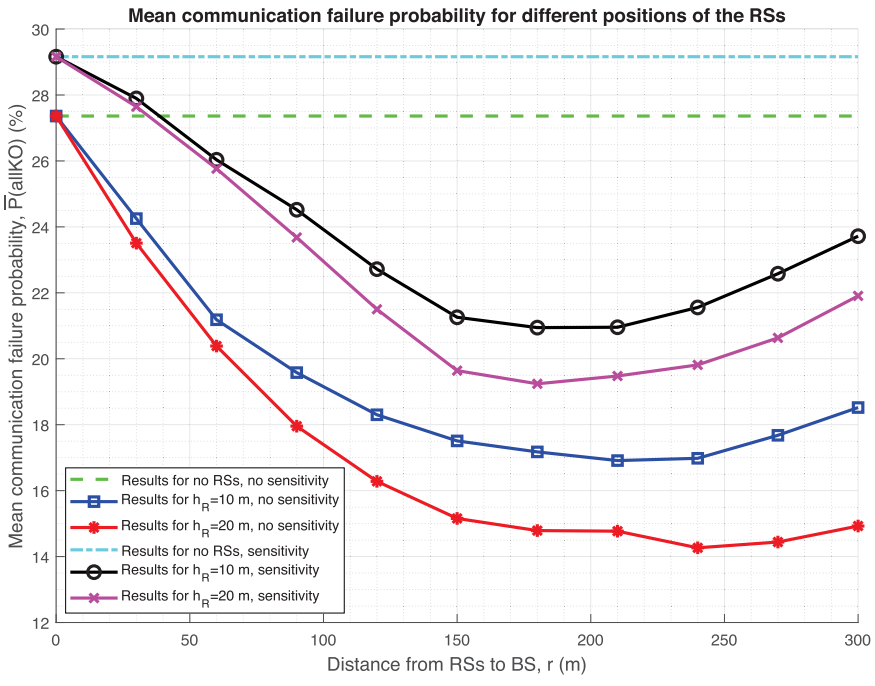

Fig. 15. Average communication failure probability depending on the position of the RSs. Power budget limitations are considered.

probability of being blocked in the RU link. This aspect should be taken into account in the relay deployment. Ideally, we want to deploy the RSs to minimize the overall probability of blockage of the users within the cell.

In any case, for successful communication, both blockage probability and link budget constraints are equally important. In LOS conditions, the link budget parameters determine the maximum allowable path loss (and additional signal losses) to prevent link failure. To get a first approximation of what the results in a real deployment could look like, we have included the link budget (i.e., transmitter power and antenna gain, including beamforming gain) and sensitivity parameters [29], summarized in Table II. These values result in a maximum available path loss of $138.2 \mathrm{~dB}$ for the BS-RS link and $122.5 \mathrm{~dB}$ for the RS-UE link. Whenever the actual path loss is greater than the maximum available path loss, the receiver (i.e., relay or UE depending on the link considered) will not be able to decode the signal correctly, even in LOS conditions.

Accordingly, in Fig. 15 we plot the average communication failure probability due to blocking only and due to blocking and limited link budget, for different values of the distance $r$ between the BS and the RSs and their heights $h_{R}$. This probability is averaged w.r.t. the random position of the user. The sizes of the blocking elements are distributed according to $L \sim \mathcal{U}\left[0, L_{\max }\right], W \sim \mathcal{U}\left[0, W_{\max }\right], H \sim \mathcal{U}\left[0, H_{\max }\right]$, and $\Theta \sim \mathcal{U}\left[0, \Theta_{\max }\right]$ (see the maximum values in Table II). We also include the case when RSs are not deployed, obtained from (32).

Some important conclusions that the figure raises is that the position of the RSs where $\overline{\mathbb{P}}(\operatorname{all} K O)$ is minimum is not at half of the radius of the cell (that would be at $150 \mathrm{~m}$ in this case) but somehow closer to the edges of the cell, which is consistent with the results shown in Fig. 14. Moreover, it should be highlighted that considering the sensitivity and power constraints has a high impact on performance and deployment. Firstly, it is shown that, when including the sensitivity in the analysis, the communication failure probability increases by more than $5 \%$. Secondly, the distance $r$ from the BS to the RS where the minimum of communication failure probability is achieved is slightly reduced when compared to the situation in which sensitivity and power constraints are not taken into account.

\section{SUMMARY AND CONCLUSIONS}

In this paper, we have considered different models for blocking elements and, in particular, a 3D model with a rectangular base and a finite height. This is the model that best fits actual buildings in urban environments.

Then, we have derived the expression of the probability of blockage for $N$ different links while taking into account the statistical dependence of the blocking elements of each link. As we have checked through different analytic expressions and simulations, the correlation effect is not negligible and must be taken into account.

Finally, we have applied the obtained expressions to a cell with multiple relays and evaluated the impact of the relay positions on the average probability of communication failure within the cell. Note that failure can happen due to blockage effects and also due to insufficient signal power levels. Accordingly, we have considered maximum power constraints and sensitivity parameters, so we better approach the real scenario that such cellular deployments should face.

As the next steps, it could be interesting to analyze both analytically and with field testing a real scenario and make a comparison between them to check how the derived expressions fit reality. The inclusion of a factor in terms of signal level reduction to incorporate the atmospheric effects and the fading could be considered. Also, the analysis of the mobility of the users and its impact on the duration of the blockage events are left for future work.

\section{REFERENCES}

[1] J. G. Andrews, T. Bai, M. N. Kulkarni, A. Alkhateeb, A. K. Gupta, and R. W. Heath, Jr., "Modeling and analyzing millimeter wave cellular systems," IEEE Trans. Commun., vol. 65, no. 1, pp. 403-430, Jan. 2017.

[2] E. Dahlman, S. Parkvall, and J. Sköld, 5G NR: The Next Generation Wireless Access Technology. London, U.K.: Academic, 2018.

[3] T. S. Rappaport, R. W. Heath, R. C. Daniels, and J. N. Murdock, Millimeter Wave Wireless Communications. Upper Saddle River, NJ, USA: Prentice-Hall, 2015.

[4] R. W. Heath, N. Gonzalez-Prelcic, S. Rangan, W. Roh, and A. M. Sayeed, "An overview of signal processing techniques for millimeter wave MIMO systems," IEEE J. Sel. Topics Signal Process., vol. 10, no. 3, pp. 436-453, Apr. 2016.

[5] T. S. Rappaport et al., "Millimeter wave mobile communications for 5G cellular: It will work!," IEEE Access, vol. 1, pp. 335-349, 2013.

[6] A. K. Gupta, J. G. Andrews, and R. W. Heath, "Macrodiversity in cellular networks with random blockages," IEEE Trans. Wireless Commun., vol. 17, no. 2, pp. 996-1010, Feb. 2018.

[7] K. Venugopal and R. W. Heath, "Millimeter wave networked wearables in dense indoor environments," IEEE Access, vol. 4, pp. 1205-1221, 2016.

[8] T. Bai, R. Vaze, and R. W. Heath, "Using random shape theory to model blockage in random cellular networks," in Proc. Int. Conf. Signal Process. Commun. (SPCOM), Jul. 2012, pp. 1-5.

[9] T. Bai, R. Vaze, and R. W. Heath, "Analysis of blockage effects on urban cellular networks," IEEE Trans. Wireless Commun., vol. 13, no. 9, pp. 5070-5083, Sep. 2014.

[10] X. Liu, J. Xu, and H. Tang, "Analysis of frequency-dependent line-ofsight probability in 3-D environment," IEEE Commun. Lett., vol. 22 no. 8, pp. 1732-1735, Aug. 2018.

[11] D. Stoyan, W. Kendall, and J. Mecke, Stochastic Geometry and its Applications, 2nd ed. Hoboken, NJ, USA: Wiley, 1995. 
[12] F. Baccelli and B. Blaszczyszyn, Stochastic Geometry and Wireless Networks, Theory. vol. 1, Delft, The Netherlands: NOW, 2009.

[13] M. Haenggi, Stochastic Geometry for Wireless Networks. Cambridge, U.K.: Cambridge Univ. Press, 2013.

[14] R. Cowan, "Objects arranged randomly in space: An accessible theory," in Advances in Applied Probability. Sheffield, U.K.: Applied Probability Trust, 1989. [Online]. Available: http://www.jstor.org/stable/1427635

[15] A. K. Gupta, J. G. Andrews, and R. W. Heath, "Impact of correlation between link blockages on macro-diversity gains in mmWave networks," in Proc. IEEE Int. Conf. Commun. Workshops (ICC Workshops), May 2018, pp. 1-6.

[16] E. Hriba and M. Valenti, "Correlated blocking in mmWave cellular networks: Macrodiversity, outage, and interference," Electron., vol. 8, no. 1187 , pp. 1-17, Oct. 2019.

[17] Y. Liu, Y. Jian, R. Sivakumar, and D. M. Blough, "Optimal access point placement for multi-AP mmWave WLANs," in Proc. 22nd Int. ACM Conf. Model., Anal. Simul. Wireless Mobile Syst. MSWIM, 2019, pp. 35-44.

[18] K. Belbase, Z. Zhang, H. Jiang, and C. Tellambura, "Coverage analysis of millimeter wave decode-and-forward networks with best relay selection," IEEE Access, vol. 6, pp. 22670-22683, 2018.

[19] S. Kwon and J. Widmer, "Relay selection for mmWave communications," in Proc. IEEE 28th Annu. Int. Symp. Pers., Indoor, Mobile Radio Commun. (PIMRC), Oct. 2017, pp. 1-6.

[20] C. Han and S. Duan, "Impact of atmospheric parameters on the propagated signal power of millimeter-wave bands based on real measurement data," IEEE Access, vol. 7, pp. 113626-113641, 2019.

[21] C. Han, Y. Bi, S. Duan, and G. Lu, "Rain rate retrieval test from 25-GHz, 28-GHz, and 38-GHz millimeter-wave link measurement in beijing," IEEE J. Sel. Topics Appl. Earth Observ. Remote Sens., vol. 12, no. 8, pp. 2835-2847, Aug. 2019.

[22] Y. Niu, Y. Li, D. Jin, L. Su, and A. V. Vasilakos, "A survey of millimeter wave communications (mmWave) for 5G: Opportunities and challenges," Wireless Netw., vol. 21, no. 8, pp. 2657-2676, Nov. 2015

[23] R. W. Heath, M. Kountouris, and T. Bai, "Modeling heterogeneous network interference using Poisson point processes," IEEE Trans. Signal Process., vol. 61, no. 16, pp. 4114-4126, Aug. 2013.

[24] A. Papoulis and S. U. Pillai, Probability, Random Variables and Stochastic Processes. New York, NY, USA: McGraw-Hill, 2002.

[25] G. Modica and L. Poggliolini, A First Course in Probability and Markov Chains. Hoboken, NJ, USA: Wiley, 2012.

[26] N. Wei, X. Lin, and Z. Zhang, "Optimal relay probing in millimeterwave cellular systems with device-to-device relaying," IEEE Trans. Veh. Technol., vol. 65, no. 12, pp. 10218-10222, Dec. 2016.

[27] Y. Yan, Q. Hu, and D. M. Blough, "Path selection with amplify and forward relays in mmWave backhaul networks," in Proc. IEEE 29th Annu. Int. Symp. Pers., Indoor Mobile Radio Commun. (PIMRC), Sep. 2018.

[28] Q. Hu and D. M. Blough, "Relay selection and scheduling for millimeter wave backhaul in urban environments," in Proc. IEEE 14th Int. Conf. Mobile Ad Hoc Sensor Syst. (MASS), Oct. 2017.

[29] 5G Millimeter Wave Frequencies and Mobile Networks: A Technology Whitepaper on Key Features and Challenges (Version 2), International Wireless Industry Consortium (IWPC), Doylestown, PA, USA, Oct. 2019.

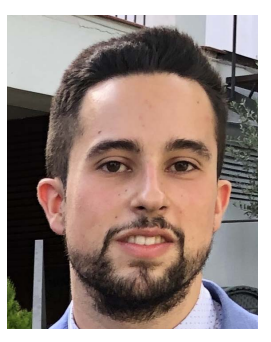

Cristian García Ruiz was born in Badalona, Spain, in 1996. He received the B.Sc. and M.Sc. degrees from the Universitat Politècnica de Catalunya (UPC), Barcelona, in 2018 and 2020, respectively, where he is currently pursuing the master's degree in railway system and electrical drive. He is set to take his Ph.D. studies at the Signal Theory and Communications Department, in 2021.

From June 2017 to April 2018, he was with Cellnex Telecom at the Broadcast Engineering and Provision Department, working on the support of technical projects for broadcasting centers in Spain. He is currently working at Sener Ingeniería y Sistemas at the Intelligent Systems for Transport Infrastructures Section, mainly developing communication and railway signaling projects for railway systems among others. His current research interests include mmWave propagation modeling, signal propagation in tunnels, mobile communications in railway systems, beamforming techniques, array processing, array pattern synthesis, massive multiple-input multiple-output channels, stochastic geometry, and 5G network-slicing.

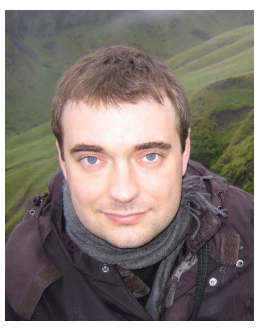

Antonio Pascual-Iserte (Senior Member, IEEE) was born in Barcelona, Spain, in 1977. He received the M.Sc. degree in electrical engineering and the $\mathrm{Ph} . \mathrm{D}$. degree from the Universitat Politècnica de Catalunya (UPC), Barcelona, in 2000 and 2005, respectively.

From September 1998 to June 1999, he was a Teaching Assistant in the field of microprocessor programming with the Department of Electronic Engineering, UPC. From June 1999 to December 2000, he was with Retevision R\&D, working on the implantation of the DVB-T and T-DAB networks in Spain. In January 2001, he joined the Department of Signal Theory and Communications, UPC, where he worked as a Research Assistant until September 2003. He received a pre-doctoral grant from the Catalan government for his Ph.D. studies during this period. He became an Assistant Professor in September 2003 and an Associate Professor in April 2008. He was also collaborating on a regular basis with the Centre Tecnològic de Telecomunicacions de Catalunya (CTTC) from October 2005 to September 2012. He currently teaches undergraduate courses in linear systems, communications, and signal theory. He also teaches postgraduate courses on advanced signal processing with the Department of Signal Theory and Communications, UPC. He has been involved in several research projects funded by the Spanish Government and the European Commission. He has also published several papers in international and national conference proceedings and journals. His current research interests include array processing, robust designs, orthogonal frequency-division multiplexing, multiple-input multiple-output channels, multiuser access, stochastic geometry, 5G network-scale performance evaluation, massive machine-type communications, mmWave transmission modeling, and optimization theory.

Dr. Pascual-Iserte received the First National Prize of 2000/2001 University Education from the Spanish Ministry of Education and Culture and the Best 2004/2005 Ph.D. Thesis Prize from UPC.

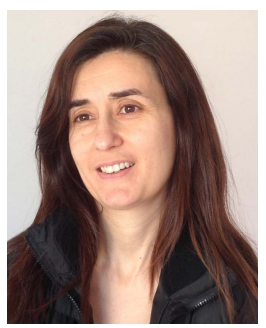

Olga Muñoz (Member, IEEE) received the M.S. and $\mathrm{Ph} . \mathrm{D}$. degrees in electrical engineering from the Universitat Politècnica de Catalunya (UPC), Spain, in 1993 and 1998, respectively. In 1994, she joined the Department of Signal Theory and Communications, UPC, where she teaches graduate and undergraduate courses related to signal processing and communications. She has been a Visiting Associate Professor at Stanford University in September-November 2014 and January-June 2015, respectively. She has served as a Reviewer for the Spanish Research Council. Besides, she has also served as a Reviewer in numerous journals and conferences. She accumulates a substantial experience in relaying and cooperative upgraded networks backed by her work on European Commission projects ROMANTIK (5thFP), FIREWORKS (6thFP), and ROCKET (7thFP). She also has experience in heterogeneous and femtocell based networks backed by her work in the project FREEDOM (7thFP), and the Spanish Government funded project MOSAIC (call 2010). She has participated in TROPIC (7thFP), pushing the idea of merging cloud computing with femtocell networking, and in TUCAN3G (7thFP) focused on providing connectivity to rural areas through new wireless technologies for the access network and WiLD (WiFi for Long Distances)-WiMAXVSAT heterogeneous backhauling. More recently, she has been working on designing, analyzing, and evaluating radio technologies in ultra-dense networks to meet the requirements for capacity and quality of service, distributed intelligence, and flexibility needed for the 5G and beyond. She has published over 50 papers in books, international conferences, and journals in the areas of signal processing and communications. 